Submitted to

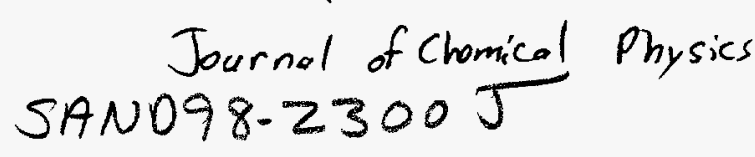

\title{
Tethered chains in poor solvent conditions: An experimental study \\ involving Langmuir diblock copolymer monolayers
}

\author{
M.S. Kent \\ Sandia National Laboratories, Albuquerque, NM. \\ J. Majewski, G. S. Smith \\ Los Alamos National Laboratories, Los Alamos, NM. \\ L. T. Lee \\ Lab. Leon Brillouin, CEN-Saclay, 91191 Gif-sur-Yvette Cedex, France \\ S. Satija \\ NIST Center For Neutron Research \\ National Institute of Standards and Technology, Gaithersburg, MD.
}

\begin{abstract}
We have employed Langmuir monolayers of highly asymmetric polydimethylsiloxanepolystyrene (PDMS-PS) diblock copolymers on dioctyl phthalate (DOP) at temperatures ranging from $22{ }^{\circ} \mathrm{C}$ to $-35^{\circ} \mathrm{C}$ as a model system for tethered chains in poor solvent conditions. The thicknesses of the tethered PS layers extending into the DOP subphase, measured by neutron reflection, decrease with decreasing temperature $(\mathrm{T})$ over this entire range. However, the variation with $\mathrm{T}$ becomes weak below $-20^{\circ} \mathrm{C}$. At the lowest $\mathrm{T}$, the layer thicknesses are contracted $55 \%-75 \%$ of their values at the theta condition $\left(\mathrm{T}_{\theta}=\right.$ $22^{\circ} \mathrm{C}$ ), but are still quite swollen compared to the fully collapsed, nonsolvent limit. The contraction of the layer with decreasing $T$ is determined as a function of surface density and molecular weight. These data are compared to universal scaling forms. The PS segments are depleted from the air surface over the entire $T$ range, the thickness of the depletion layer increasing slightly with decreasing $T$. The free energy of the surface layer is probed by surface tension measurements. Negative surface pressures are observed at low coverages for both PDMS-PS and PDMS monolayers, indicating metastability toward lateral phase separation. Evidence for a transition from a dispersed phase to a condensed phase with decreasing $T$ was observed in the reflectivity at very low PDMS-PS coverage. At high coverage where the submerged blocks are strongly interacting at $22^{\circ} \mathrm{C}$, only a modest decrease in surface pressure is observed over the experimental range of $\mathrm{T}$. This latter iresult is discussed in terms of the relative contributions of enthalpic and entropic effects to the surface pressure.

This work was partially supported by the U.S.SDepartment of Energy under contracts DE AC04-94AL85000 and W-7405-ENG 36 .
\end{abstract}




\section{DISCLAIMER}

This report was prepared as an account of work sponsored by an agency of the United States Government. Neither the United States Government nor any agency thereof, nor any of their employees, make any warranty, express or implied, or assumes any legal liability or responsibility for the accuracy, completeness, or usefulness of any information, apparatus, product, or process disclosed, or represents that its use would not infringe privately owned rights. Reference herein to any specific commercial product, process, or service by trade name, trademark, manufacturer, or otherwise does not necessarily constitute or imply its endorsement, recommendation, or favoring by the United States Government or any agency thereof. The views and opinions of authors expressed herein do not necessarily state or reflect those of the United States Government or any agency thereof. 


\section{DISCLAIMER}

Portions of this document may be illegible in electronic image products. Images are produced from the best available original document. 


\section{Introduction}

Polymer chains which are fixed (or tethered) by one end to a surface or interface have been extensively investigated in recent years. ${ }^{1-4}$ On the practical side, they are a useful means of modifying properties to control adhesion, ${ }^{5}$ protein adsorption, ${ }^{4,6}$ or the attraction between colloidal particles. ${ }^{7}$ In addition, they are an interesting system for examining the physics of polymers in confined geometries, and the balance of enthalpic and entropic contributions to the free energy. Such layers can be formed or employed in a variety of solution conditions and geometries. The present article concerns planar layers of tethered chains in solvents whose solvating power is poorer than the theta condition. For this case, it is now recognized that with decreasing solvent quality, important changes in segment density distribution can occur both normal and lateral to the tethering surface. This behavior can contribute to the understanding of certain features of biological systems, such as protein folding. Solvent quality can be varied either through the choice of solvent or by changing the temperature (T). The present study employs the latter method. The main points of focus are the manner in which the layers contract with decreasing solvent quality, the lateral phase behavior, and the free energy within the tethered layer.

The behavior of tethered chains in poor solvent conditions has been addressed theoretically by scaling, ${ }^{8.9}$ analytic ${ }^{10}$ and numerical ${ }^{11-13}$ self-consistent field (SCF) calculations, the random phase approximation combined with a numerical mean-field analysis, ${ }^{14}$ a single-chain-mean-field (SCMF) statistical mechanical approach, ${ }^{2.15 .16}$ and Monte Carlo (MC) $)^{17-20}$ and molecular dynamics (MD) simulations. ${ }^{21}$ An important distinction in these studies is whether the tethering points are fixed or have mobility in the plane of the surface, as this plays a critical role in the lateral phase behavior. A further point of distinction for the case of fixed tethering points is whether the tethering points are random or regular. ${ }^{13,14,17}$ 
The case of fixed tethering points was initially treated by scaling ${ }^{8.9}$ and analytical SCF methods. ${ }^{10}$ These approaches assumed lateral uniformity within the layer, and addressed variations in the free energy and the segment concentration profile normal to the surface. The principal focus was the nature of the contraction of the layer with decreasing solvent quality, and how the dependence of layer height on solvent quality varies with surface density $(\sigma)$. Two limits were discussed. The contraction of isolated, noninteracting tethered coils is expected to be analogous to the contraction of free chains in dilute solution..$^{22-24}$ On the other hand, in dense layers such that the chains are strongly stretched, the above approaches showed that interchain interactions shift the contraction to lower temperatures and broaden the temperature range. Numerical SCF and simulation studies have addressed the behavior in between these two limits. ${ }^{13.17 .18,21}$ For real chains, the contraction is expected to be continuous for all surface densities due to their finite molecular weight. The degree of interaction among the tethered chains can be compared for different molecular weights using a reduced surface density $\Sigma\left(=\sigma \pi R_{B}{ }^{2}\right.$ where $R_{g}$ is the dilute solution free chain radius of gyration).

Various studies have addressed the lateral structure as well as the contraction normal to the surface. ${ }^{13-21}$ Lai and Binder originally showed by MC simulation that for chains with fixed tethering points, a decrease in solvent quality leads to lateral fluctuations in segment density, in addition to contraction of the layer normal to the surface. ${ }^{17}$ A similar effect was subsequently observed in MD simulations by Grest and Murat. ${ }^{21 a}$ Yeung et al. studied lateral aggregation of chains in relatively dense tethered layers by combining a numerical mean-field analysis with the random phase approximation. ${ }^{14}$ In particular, they found that the fluctuations are restricted to the outer edge of the layer for higher molecular weights and surface densities, but exist throughout the thickness of the layer at lower surface densities and molecular weights. This was subsequently confirmed by $2-D$ numerical SCF calculations. ${ }^{13}$ Williams used a scaling approach to address the regime of moderate grafting densities (between isolated chains 
and the strong stretching limit) in the nonsolvent limit. ${ }^{25}$ Kinetic effects were considered for the case of a rapid change in solvent quality. In particular, surface micelles were predicted for the case of a sudden decrease in solvent quality and sufficiently high molecular weight chains such that they are unable to disengage and become isolated prior to the aggregation transition.

For chains with tethered ends which are mobile, as in the present experimental system, a lateral phase transition into polymer-rich and polymer-poor phases is predicted. Detailed isotherms and phase diagrams including regions of metastability have been calculated by Szleifer et al. ${ }^{2.15,16}$ The present data will be discussed with respect to this lateral phase transition, in addition to the contraction normal to the surface.

Previous experimental work on tethered chains in poor solvent conditions can also be distinguished by the mode of tethering. Perahia et al. examined PS chains which were weakly tethered to silicon via an adsorbing zwitterionic end group. The tethered layers were immersed in cyclohexane $\left(\mathrm{T}_{\theta}=34^{\circ} \mathrm{C}\right) .^{26}$ As a consequence of the weak anchoring energy, the surface density of adsorbed chains varied with temperature. The analysis focussed on the equilibrium between chains in the bulk and in the tethered layer. At high bulk concentrations, a wetting layer of adsorbed chains was present in addition to the tethered layer.

The case of chains which are strongly tethered to a surface with fixed tethering points has been examined by Auroy et al., ${ }^{27,28}$ Karim et al., ${ }^{29,30}$ and more recently by Gallagher et al., ${ }^{31}$ and Baker et al. ${ }^{32}$ In this case, the chains do not desorb on experimental time scales. Auroy et al., examined PDMS chains end-grafted onto silica particles by neutron scattering. The layers were grafted over a wide range of surface density ( $\Sigma \sim 4$ to 27 ). They examined the tethered layers in methanol (nonsolvent), acetone (poor solvent), in mixtures of methanol and dichloromethane (DCM, good solvent), and over a range of temperature in styrene $\left(\mathrm{T}_{\theta, \mathrm{PDMS}} \cong 30^{\circ} \mathrm{C}\right)$. In methanol, a very dense, nearly fully collapsed layer was observed with average PDMS volume fraction of

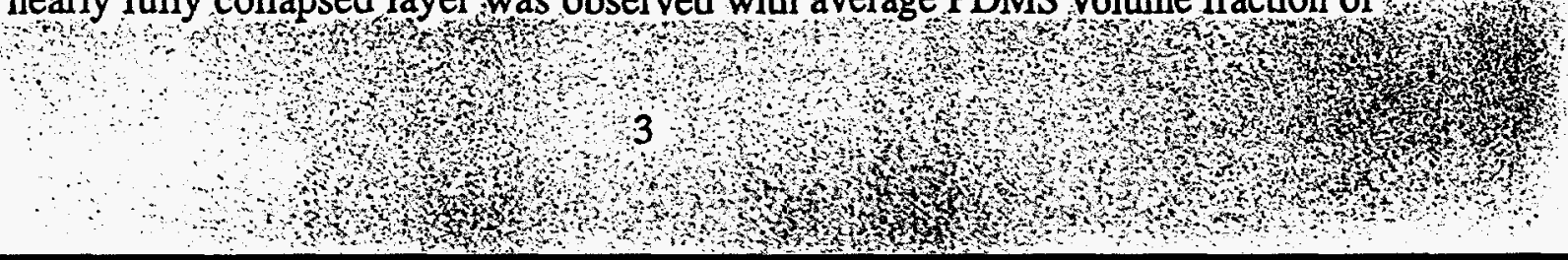


0.84. In acetone, a slightly better but still poor solvent, the same layer had an average PDMS volume fraction of 0.64 . For the methanol-DCM mixtures, the situation was complicated by a nonuniform distribution of solvent within the tethered layer. The expansion of the layer was not linear with increasing DCM weight fraction, but rather most of the expansion occurred over a fairly narrow range of composition (65\% to 80\% DCM). The transition was much broader at lower surface density. This latter result is contrary to expectation, and was attributed to preferential solvation. In styrene, they reported a linear variation of layer thickness with $\mathrm{T}$ over the entire range of reduced temperature $\tau\left(=\left(T-T_{\theta}\right) / T\right.$, where $T_{\theta}$ is the theta temperature) from 0.11 to -0.17 . Little detail was obtained about the profile, as a single step model was used in the analysis of the scattering data.

Karim et al. studied PS chains end-grafted onto silicon and immersed in cyclohexane by neutron reflection. The detailed form of the profile was examined for temperatures ranging down to $9.5^{\circ} \mathrm{C}(\tau=-0.09)$. The layer was grafted at relatively high surface density $(\Sigma \cong 17)$. They found good agreement with the analytic SCF profile of Zhulina et al. ${ }^{10}$ at $\mathrm{T}_{\theta}$. In particular, the decrease in $\phi$ with depth in the body of the profile followed the functional form $\phi(z)=\phi_{0}\left[1-\left(z / L_{0}\right)^{2}\right]^{y}$, with an exponent $y$ of $1 / 2$. At $9.5^{\circ} \mathrm{C}$, the maximum volume fraction was 0.47 , still far from that of the fully collapsed state. The profile exponent $y$ seemed to be levelling off at the lowest $\mathrm{T}$ at a value of roughly 1/3. No depletion layer was reported, but rather the dangling chains collapsed onto the surface. The magnitude of the tail of the profile decreased with decreasing $T$. A sigmoidal shape was obtained for the contraction of the tail with $\tau$, which indicates swelling analogous to that for dilute free chains. The main body of the profile was reported to swell in a different manner than the tail, being more like that observed for crosslinked gels.

The lateral structure of tethered chains in poor solvent and nonsolvent conditions has been examined by AFM imaging. 33,36 . Tethered chain systems have been imaged in H. 
both small molecule liquids, and also in air. The results in nonsolvent conditions indicate lateral heterogeneity at low and intermediate surface densities. Images show a nearly uniform appearance at high grafting densities, while isolated single chains are observed at low grafting densities. It is not clear whether AFM can resolve lateral heterogeneity occuring only in the tip of an otherwise uniform brush, as predicted for high densities by Yeung et al. ${ }^{14}$ Also, the value of $\tau$ at which lateral clumping begins to occur when the solvent quality is decreased below the theta condition has not yet been established. ${ }^{36}$

Below we report results for a system in which chains are strongly tethered to a surface, such that they do not desorb, but yet have mobility in the plane of the surface. This system is composed of Langmuir monolayers of highly asymmetric polydimethysiloxane-polystyrene (PDMS-PS) diblock copolymers on the surface of an organic liquid. The PDMS block anchors the copolymers to the surface due to the low surface energy of PDMS relative to the subphase liquid, while the (fully deuterated) PS block dangles into the subphase liquid. The segmental concentration profile of the d-PS block is examined by neutron reflection. The neutron scattering length density of the PDMS block is nearly matched with that of air, and does not contribute to the reflectivity. The copolymers are sufficiently asymmetric such that interchain interactions occur nearly entirely through the PS blocks. Using such systems, we reported previously on the structure and free energy of monodispersed tethered chains in good and theta solvent conditions $^{37-40}$ and in solutions of free chains, ${ }^{41-42}$ the surface rheological properties of tethered chains in good solvents, ${ }^{43}$ and the structure of tethered layers with bimodal molecular weight distributions. ${ }^{44}$

In the present report we examine the poor solvent regime using dioctylphthalate (DOP) as the subphase liquid. The theta condition for PS in DOP occurs at $22^{\circ} \mathrm{C} .{ }^{45}$ The accessible range of temperature with this system is ultimately limited by the freezing of $D O P$ at $-50^{\circ} \mathrm{C}$. In this work we have examined the structure of the tethered layer from $22^{\circ} \mathrm{C}$ to $-35^{\circ} \mathrm{C}$, corresponding to a range of $\tau$ from 0 to 0.25 . This range extends much

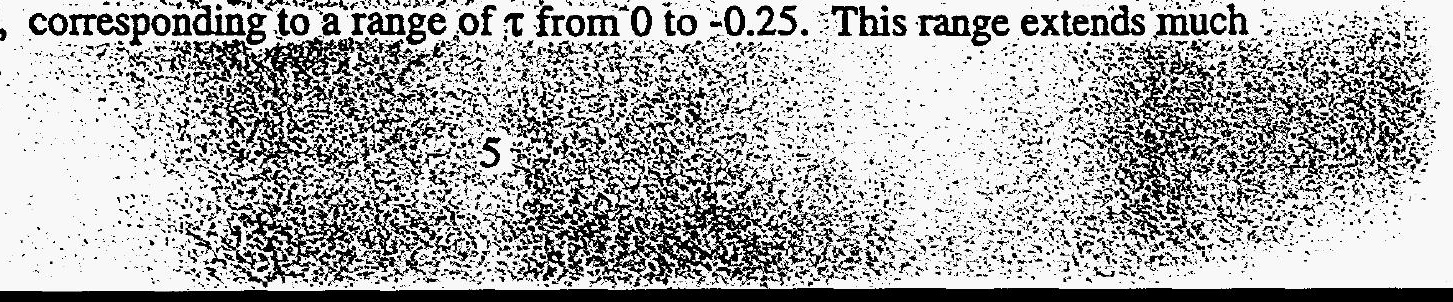


further below $T_{\theta}$ than in previous studies. Another distinction of the present system is that the surface density and molecular weight are varied independently. Our previous work has shown that the asymptotic strong stretching limit is not achieved in good or theta solvents within the range of surface density accessible to this system $(\Sigma=1-11)$. Thus, the present study addresses the intermediate surface density regime between isolated tethered chains and dense brushes. The surface tension of DOP is far lower than that of PS for the entire range of temperatures examined, thus the air surface is repulsive for the dangling d-PS blocks. The strongly repulsive nature of the interface for the dangling block is a further important distinction from previous work. Another study of a Langmuir diblock copolymer monolayer system, but with a polyelectrolyte dangling block, has been reported recently. ${ }^{46}$

In addition to examining the structure of the layer normal to the surface by neutron reflection, the interactions among the copolymers within the monolayer and the lateral phase behavior are examined through surface tension measurements. The surface pressure $\left(\Pi=\gamma_{0}-\gamma\right.$, where $\gamma$ and $\gamma$ are the surface tensions of the pure solvent and monolayer-covered surface, respectively) is related to the free energy $(F)$ of the monolayer by $\Pi=-(\partial F / \partial A)_{\text {n.T.P. }}$, where $A$ is the surface area and $n$ is the number of copolymer molecules. We examine the increase in surface tension with decreasing temperature for pure DOP, and for DOP surfaces with PDMS and PDMS-PS monolayers. At lower coverages, negative surface pressures are observed with decreasing temperature, which indicate metastability. At higher $\Sigma$ only positive pressure is observed, and we examine the variation in surface pressure with decreasing $\mathrm{T}$ in order to gain insight into enthalpic and entropic contributions in layers of interacting tethered chains. In our previous work in good and theta solvent conditions, the maximum attainable surface density was limited by a sharp rise in surface pressure caused by the interactions of the submerged blocks. ${ }^{39,40}$ The origin of this shap rise in pressure has been puzzling, and remains an important focus of the present report. 
The paper is organized as follows. Following a description of the experimental details, the results are presented in two sections. In Section III A, we describe the form of the segmental concentration profile normal to the surface, and the contraction of the layer with decreasing $T$ for a range of both $\sigma$ and $M$. In Section III $B$, we report the surface pressure isotherms for PDMS-PS and PDMS monolayers as a function of T. In Section IV, which is divided into the two corresponding sections, the data are discussed in light of previous experimental and theoretical work. The paper concludes with a brief summary of the most important conclusions in Section V.

\section{Experimental}

\section{A. Materials}

The two PDMS-PS diblock copolymers used in this study are denoted 20-170 and 28-330, where the labels represent the molecular weights (in $\mathrm{kg} / \mathrm{mol}$ ) of the PDMS and PS blocks, respectively. The PS blocks are fully deuterated. These samples were described in a previous report. ${ }^{39}$ A PDMS homopolymer $\left(25,800 \mathrm{~g} / \mathrm{mol}, \mathrm{M}_{w} / \mathrm{M}_{n}=1.03\right)$ used for isotherm measurements was purchased from Polymer Standards Service (FRG). ${ }^{47}$ Dioctyl phthalate was obtained from Aldrich and further purified before use following the method outlined by Balsara et al..$^{48}$ Chloroform obtained from Aldrich was used as received for spreading the copolymer monolayers.

\section{B. Procedures}

A schematic diagram of the Langmuir trough used in the neutron reflectivity study is shown in Figure 1. It has internal dimensions of $75 \mathrm{~mm} \times 152 \mathrm{~mm} \times 4 \mathrm{~mm}$, and was constructed from a solid Teflon block. Beneath the liquid the Teflon was very thin, approximately $0.3 \mathrm{~mm}$, which facilitated heat transfer. To minimize temperature gradients within the liquid and at the air surface, a Plexiglas cover was placed over the trough. The cover contained $2 \mathrm{~mm}$ thick guartz windows for the neutron beam, and 
openings for a thermocouple and the surface tensiometer. The latter was mounted on the top of the Plexiglas cover. The trough was bolted to a copper plate and was cooled by three thermoelectric Peltier elements (Melcor). One face of each element was in contact with a thick copper block through which a cooling fluid was circulated. The second face of each Peltier element was in contact with the copper plate attached to the trough. A thin layer of thermally conductive grease was applied between between all surfaces in contact to aid heat transfer. The entire set-up was enclosed in a canister which was purged with dry argon to remove water vapor. Amorphous quartz windows, $2 \mathrm{~mm}$ thick, were mounted in the side walls of the canister to allow for passage of the neutron beam. During the reflectivity measurements, the surface pressure was measured with a NIMA model 9000 tensiometer using filter paper. We note that there was negligible variation in surface pressure during the period in which the reflectivity was collected $(\sim 3 \mathrm{hrs})$. The canister was placed on an active vibration isolation table to reduce external vibrations. The temperature of the subphase was measured by a thermocouple inside a stainless steel tube which was submerged into the liquid. The lateral and vertical variation in temperature measured at $5^{\circ} \mathrm{C}$ (in water) were less than $0.2^{\circ} \mathrm{C}$ and $1^{\circ} \mathrm{C}$, respectively.

For the reflectivity experiments, the copolymer monolayers were spread from dilute solution in chloroform, a mutual good solvent for PDMS and PS, using a Hamilton microsyringe. Just prior to spreading the monolayer, the bare DOP surface was aspirated to remove surface active impurities. Reflectivity measurements were performed on the NG7 reflectometer (NIST). A fixed wavelength of $4.1 \AA$ was used. The treatment of the data was the same as in our previous report for theta conditions. ${ }^{40}$ In the analysis of the reflectivity data, the roughness at the air interface was fixed at $3 \AA$. The neutron scattering length densities of d-PS, PDMS, and DOP are $6.476 \times 10^{-6}, 0.064 \times 10^{-6}$, and $0.62 \times 10^{-6} \AA^{-2}$, respectively. The copolymer surface density was determined by integrating the profiles obtained from the reflectivity. 
Surface pressure isotherms for PDMS homopolymer were measured as a function of temperature in the same trough. The surface density was determined from the volume of solution deposited onto the surface. To determine surface pressure isotherms for $\mathrm{T}<22^{\circ} \mathrm{C}$, an initial quantity of a dilute solution of PDMS in chloroform was deposited onto the surface at $22{ }^{\circ} \mathrm{C}$, and then the trough was slowly cooled. The surface tension was recorded when the system stabilized at each temperature. Following measurement at the lowest temperature, the trough was allowed to warm to $22^{\circ} \mathrm{C}$. An additional quantity of PDMS was then deposited and the process was repeated. A hysteresis in surface tension was observed for both PDMS and PDMS-PS monolayers as the temperature of the trough increased from the lowest value. However, the surface tension during the cooling cycle was reproducible following a delay period of $\sim 12$ hours after warming to $22^{\circ} \mathrm{C}$.

\section{Results}

\section{A. Segmental concentration profile}

Reflectivity data from the copolymer monolayers over a range of temperature from $22{ }^{\circ} \mathrm{C}$ to $-35^{\circ} \mathrm{C}$ are shown in Figures $2 \mathrm{a}-2 \mathrm{~d}$. Data for two surface densities for the 20-170 copolymer are shown in Figures $2 a$ and $2 b$, and for the 28-330 copolymer in Figures $2 c$ and $2 d$. The data are expressed as $\log R / R_{0}$ vs $q_{\text {, where }} R_{o}$ is the calculated reflectivity from the bare DOP surface and $q=4 \pi \sin \theta \lambda \lambda$ where $\theta$ is the angle of incidence and $\lambda$ is the wavelength. Data for different temperatures have been shifted on the vertical scale for clarity. With decreasing temperature three general trends are observed in the data: an increase in $R / R_{0}$, a shift of the maximum to higher $q$ values and a broadening of the peak, and the emergence of a minimum following the initial peak. No variation in reflectivity is detected over this temperature range for the bare DOP surface as shown in Figure 2e.

The curves through the data in Figures $2 \mathrm{a}-2 \mathrm{~d}$ are best-fits corresponding to the $\mathrm{d}$ PS volume fraction profiles $(\phi(z)$, where $z$ is the depth from the surface) in Figures $3 a-3 \mathrm{~d}$.

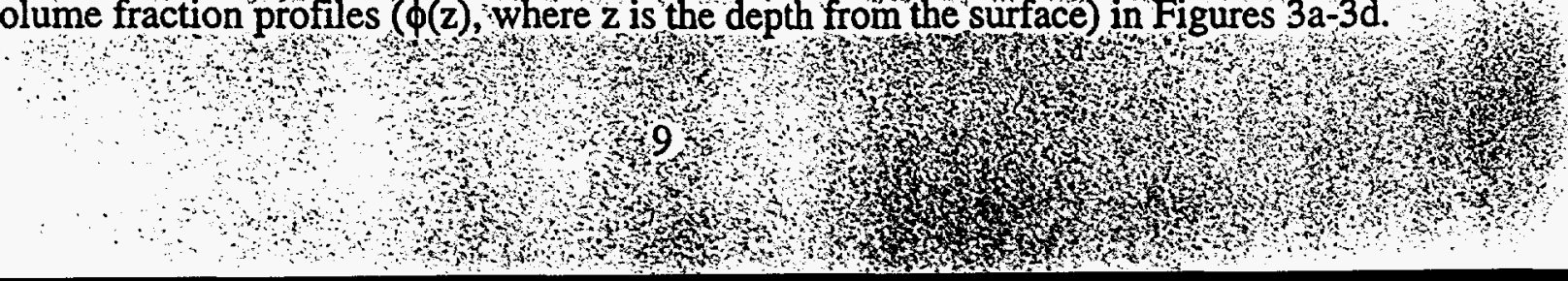


The empirical functional form used in the fitting is composed of a depletion layer, followed by a step of constant $\phi$, then followed by a smooth decrease in $\phi$ over the remainder of the profile. The rise to the step and the decrease from the step are both modelled by Gaussian functions. This model profile is the simplest form we were able to find which describes the data over the entire range of temperatures.

A depletion layer is present at all $\mathrm{T}$ and $\sigma$. We define the magnitude of the depletion layer as the distance from the air surface to the leading edge of the step. Over the experimental temperature range, the depletion layer increases from $47 \AA$ to $65 \AA$ for the 20-170 copolymer at the higher $\sigma$, and from $57 \AA$ to $81 \AA$ for the $28-330$ copolymer at the higher $\sigma$. The variation is somewhat smaller for the monolayers at lower $\sigma$, increasing from $42 \AA$ to $52 \AA$ for the $20-170$ copolymer, and from $63 \AA$ to $73 \AA$ for 28 330 copolymer.

We note that the reflectivity data can be fit without a depletion layer if very large roughnesses at the air surface are included in the model profile. For the higher $\sigma$ monolayers, such roughness values range from $16 \AA$ to $21 \AA$ for the $20-170$ copolymer and from $19 \AA$ to $28 \AA$ for the $28-330$ copolymer over the experimental T range. These values are much larger than can be accounted for by the surface PDMS block, and appear to be unphysical. These data, together with the dependencies on $\sigma$ and $M$ at $T_{\theta}$ discussed previously, ${ }^{40}$ provide strong support for the conclusion that a depletion layer is the correct interpretation.

The contraction of the layer normal to the surface is smooth for both $\sigma$ values of each copolymer monolayer. The variation with $\mathrm{T}$ of the rms layer height $\left(\mathrm{h}_{\mathrm{mms}}\right)$ and the maximum volume fraction $\left(\phi_{\mathrm{m}}\right)$ are given in Figures $4 \mathrm{a}$ and $4 \mathrm{~b}$, normalized by the values at $T_{\theta}$. Over most of the $T$ range, there is a strong dependence of $h_{\text {m }}$ and $\phi_{m}$ on $T$. However, below $-20^{\circ} \mathrm{C}(\tau \cong-0.17)$ the profiles becomes only weakly dependent on $\mathrm{T}$. The decrease of $h_{r m s} / h_{\text {rms },}$ with $|\tau|$ depends on both $\sigma$ and $M$. For fixed $M$, there is a greater reduction in $h_{\text {ms }}$ and a greater increase in $\phi_{m}$, for higher $\sigma$. At fixed $\sigma$, the

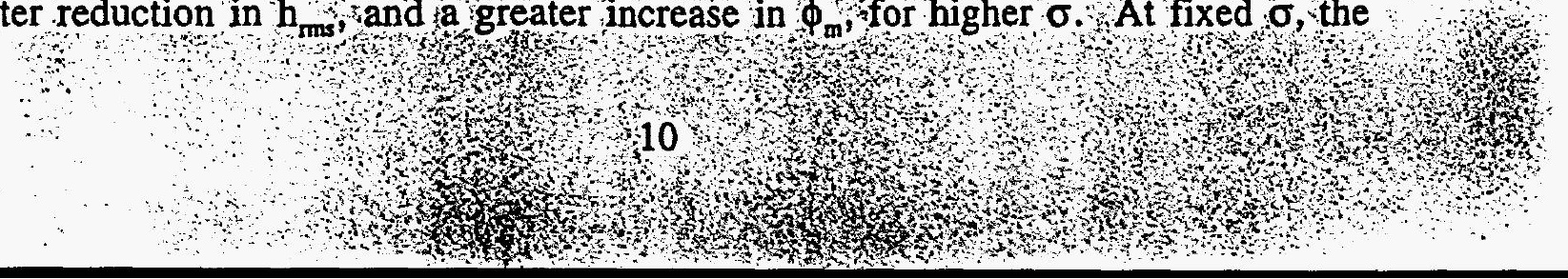


contraction is stronger for higher $M$. In Figures $4 c$ and $4 d$, the data for the layer height are plotted according to the universal scaling relations predicted for the asymptotic strong stretching limit ${ }^{10}$ and the isolated chain limit, ${ }^{23.24,49}$ respectively. In Figure $4 e$ the data are plotted according to the scaling relationship $h_{r m s} / h_{r m s, \theta} \alpha \tau / \phi_{\theta}$ where $\phi_{\theta}$ is the volume fraction of segments within the layer at the $T_{\theta}$. For a tethered layer, $\phi_{\theta}$ can be approximated by $h_{d r y} / h_{r m s, \theta}$ where $h_{d r y}$ is the dry thickness obtained by integrating the segment concentration profiles. This relationship, based on an analogy to lightly crosslinked polymer gels, ${ }^{30,50}$ adequately described the data of Karim, et al.. The comparison of the data with these universal scaling relations will be discussed further in Sec IV A.

The profiles obtained below $-20^{\circ} \mathrm{C}$ are still quite swollen with solvent compared to the fully collapsed state observed for nonsolvent conditions. ${ }^{27-31}$ The profiles for both surface densities at the lowest $\mathrm{T}$ are compared in Figure 5 for the 20-170 copolymer.

Finally, no evidence for loss of copolymer from the monolayers with decreasing temperature was observed for the series in Figure 2. The surface densities, obtained by integration of the fitted profiles, are given in Table I. No variation of surface density with $\mathrm{T}$ is observed for the lower surface densities. A small apparent increase $(\sim 5 \%)$ is observed for the $28-330$ copolymer at the higher surface density, which may be due to limited accuracy of the model functional form used in the fitting. The lack of variation of surface density with $\mathrm{T}$ is consistent with the strong anchoring of the copolymers to the surface. The anchoring energies per chain, determined from the interfacial tensions, for the $20-170$ and $28-330$ copolymers on DOP at $22{ }^{\circ} \mathrm{C}$ are $200 \mathrm{kT}$ and $266 \mathrm{kT}$, respectively. ${ }^{40}$ 


\section{B. Surface pressure}

The variation of the surface tension with temperature is plotted in Figure 6a and $6 \mathrm{~b}$ for the 20-170 and 28-330 monolayers, respectively. These data were obtained simultaneous with the reflectivity data of Figure 2. Similar data for the PDMS monolayer-covered DOP surface are shown in Figure 6c. The variation for the bare DOP surface is also shown in Figures $6 \mathrm{a}-6 \mathrm{c}$ for comparison. The surface tension increases with decreasing $\mathrm{T}$ for all surfaces, but the increase is greater for the monolayer-covered surfaces than for the bare DOP surface. Consequently, for low surface coverages, the surface tensions of the monolayer-covered surfaces exceed that of the bare DOP surface at the lowest temperatures, resulting in negative surface pressures. Negative pressures are observed for both PDMS-PS and PDMS monolayers, although the effect is much greater for the copolymer monolayers.

A negative surface pressure indicates that the surface is metastable towards lateral phase separation. Indeed, evidence for a transition from a dispersed phase to a condensed phase with decreasing temperature was observed for $\sigma$ slightly lower than the ranges in Figures $6 \mathrm{a}$ and $6 \mathrm{~b}$. This evidence was in the form of a dramatic decrease in reflectivity with decreasing T. An example of this is shown in Figure 7. For comparison, the inset in Figure 7 shows data from Figure $2 \mathrm{~b}$ obtained for the same copolymer at a slightly higher $\sigma$ (indicated by the greater maximum in $R / R_{0}$ at $22^{\circ} \mathrm{C}$ ). In the inset the reflectivity increases with decreasing $\mathrm{T}$, which is expected due to the contraction of the tethered layer. On the other hand, the decrease in reflectivity with decreasing $T$ observed in the main figure is interpreted as due to a dispersed phase/condensed phase transition. ${ }^{51}$ The original reflectivity curve was recovered upon reheating to $22^{\circ} \mathrm{C}$. The decrease in reflectivity with decreasing $\mathrm{T}$ was entirely absent for the $\sigma$ ranges of the copolymers in Figure 2, but occurred repeatedly for lower $\sigma$. We would also expect a vanishing pressure to accompany a transition from dispersed to aggregated chains. However, for 
the present system the phase transition occurs at such low $\sigma$ that the pressure in the dispersed state was too low to detect with the present transducer.

Surface pressure isotherms for the two block copolymers are compared to the isotherm for PDMS homopolymer at $22{ }^{\circ} \mathrm{C}$ and at $-30^{\circ} \mathrm{C}$ in Figures $8 \mathrm{a}$ and $8 \mathrm{~b}$, respectively. Interestingly, at fifty degrees below the theta temperature, only a modest reduction in pressure is observed relative to that at $T_{\theta}$ for the higher $\sigma$ values.

\section{DISCUSSION}

\section{A. Segmental concentration profile}

In good solvent conditions, interacting tethered chains stretch normal to the surface to reduce unfavorable segment-segment interactions..$^{1.4 .39}$ The degree of stretching is limited by entropically-based chain elasticity. With decreasing temperature/solvent quality, segment-segment interactions become less unfavorable, and the degree of stretching is reduced. At $\mathrm{T}_{\theta}$, the dimension of an isolated chain scales with $\mathrm{M}^{1 / 2}$, equivalent to that of a random walk chain. However, stretching still occurs in strongly interacting tethered layers due to higher order interactions, and the tethered chain dimension scales linearly with $M$ at sufficiently high $\sigma .^{10.21}$ Below $T_{\theta}$, segmentsegment interactions become increasingly favored relative to segment-solvent interactions. Tethered chains contract further in order to minimize interaction with the solvent. In the nonsolvent limit, previous work has shown that end-grafted tethered chains in low molecular weight liquids collapse to nearly melt density. ${ }^{27,31}$ However, the magnitude of $\left|T_{\theta}-T\right|$ at which such nonsolvent conditions are reached has not yet been established experimentally.

For the present system, the surface energy of DOP $(30.5 \mathrm{dyn} / \mathrm{cm})^{40}$ is much lower than that of the PS segments $(40.7 \mathrm{dyn} / \mathrm{cm}), 22$ and therefore the maximum in segment concentration occurs at a depth below the surface, rather than at the surface. Indeed, the 
depletion layer actually increases as the temperature decreases below $T_{\theta}$. The relationship between the segment-surface interaction and the segment concentration at the interface has been examined in detail theoretically ${ }^{11,16}$ and through MD simulations. ${ }^{21}$ The present observations of a depletion layer for a chain tethered to a repulsive surface, and the increase in its size with decreasing $T$, are consistent with the results of these studies. In contrast to the present case of a repulsive surface, a maximum in the segment concentration is predicted at the surface for an attractive segment-surface interaction.

The profiles in Figure 2 differ considerably from the profiles reported by Karim $e t$ $a l$ for PS end-grafted onto silicon and immersed in cyclohexane,${ }^{29}$ most notably in the presence of a depletion layer at the air surface. This is likely due to a different segmentsurface interaction in the two cases. In the system of Karim et al., there is a mild attraction between the PS block and the silicon substrate, and correspondingly the maximum in the segment concentration occurs at the silicon oxide surface. The higher surface density in their case may also contribute to the lack of a depletion layer. However, based on numerical SCF calculations, the effect of surface density on the depletion layer is expected to be weak over this range. ${ }^{12.53 .54}$

The contraction of the tethered layer with decreasing temperature is continuous over the experimental range of $\Sigma$. This observation is consistent with the data of Auroy et $a l^{28}$ and Karim et al ${ }^{29.30}$ for tethered chain systems at higher $\Sigma$ and fixed tethering points, and also with the contraction of free chains in dilute solution. ${ }^{49.55-58}$ The contraction of a tethered layer is predicted to occur over a broad temperature range for $\Sigma \gg 1$ due to interchain interactions within the tethered layer, but to occur over a much narrower temperature range for $\Sigma \leq 1 .^{10}$ This expected trend is not apparent in Figures $4 \mathrm{a}$ and $4 \mathrm{~b}$. The values of $\Sigma$ for the four experimental series at $22^{\circ} \mathrm{C}$ and also at $-30{ }^{\circ} \mathrm{C}$ are given in Table II. ${ }^{59}$ For all series except 20-170 at low $\sigma$, the chains are well into the interacting regime at $22^{\circ} \mathrm{C}$. While the data cover factors of $2.9(20-170)$ and $2.4(28-330)$ in $\Sigma$ at 22 ${ }^{\circ} \mathrm{C}$, these ranges are apparently too small to observe a narrowing of the temperature range 
of the contraction with decreasing $\Sigma$. The contraction is in all cases significantly broader than that for dilute $2,000 \mathrm{Kg} / \mathrm{mol}$ free PS chains in DOP reported by Stepanek et al.$^{56}$ The isolated chain limit could not be examined in detail due to lateral phase separation discussed further below. However, the data for the 20-170 copolymer at the lower $\sigma$ approaches this limit, as indicated by the fact that no stretching is observed at $T_{\theta}$ for this monolayer (Figure 4d). For this case, the contraction occurs over the same $T$ range as for the strongly interacting monolayers.

The decrease of $h_{r m s} / h_{r m s, \theta}$ with $|\tau|$ depends upon both $\sigma$ and $M$, as observed in Figure 4a. These data can be considered with respect to two limits. First, the data clearly do not follow the dependence predicted for the asymptotic strong stretching limit, as demonstrated in Figure 4c. This is expected, since our previous work has shown that this limit is only reached for $\Sigma \gg 11$ at $\mathrm{T}_{\theta}$, beyond the accessible range of $\Sigma$ for this system. Moreover, $\Sigma$ decreases with decreasing $\mathrm{T}$ as the dangling PS chains contract (see Table II).

The other limiting case is that for isolated, noninteracting chains $(\Sigma \leq 1)$ In this limit the contraction of the layer is expected to be analagous to that of free chains in dilute solution. For isolated free chains $R_{g} / R_{g, \theta}$ is a function of $k M^{1 / 2}{ }^{22-24.49 .55-58}$ For $\tau$ $\ll 0, R_{g} / R_{g, \theta} \alpha\left(k M^{1 / 2}\right)^{-1 / 3}$, which is termed the "collapsed" regime, although the chains may still be quite expanded relative to the fully collapsed, nonsolvent limit. The present data are compared to this limiting behavior in Figure $4 \mathrm{~d}$. In this figure the rms layer heights are normalized by the rms layer height at $T_{\theta}$ in the limit of $\Sigma \rightarrow 0$. This representation shows that the layers are stretched normal to the surface at $T_{\theta}$, due to interactions between the chains. ${ }^{60}$ However, as the temperature decreases the interactions among the chains become weaker and the dependence on $\Sigma$ diminishes. At the lowest temperatures, the data approach the universal scaling predicted for collapsed isolated chains in a poor solvent. The remaining small dependence on $\Sigma$ is likely due to the fact 
that the noninteracting regime is not quite reached at the lowest $T$ for the higher $\Sigma$ monolayers.

The dependence of $h_{\mathrm{ms}}$ on $\tau$ is very weak at the lowest $\tau$ values. This indicates that the systems are in the "collapsed" regime. The dependence is somewhat weaker than $\mathrm{h}_{\mathrm{mms}} \alpha \mathrm{K}^{1 / 3}$ predicted for free chains in dilute solution, as shown in the inset to Figure 4d. The difference is outside the experimental error. Error bars indicating the uncertainty in the rms layer height are shown in the inset. Uncertainty in the temperature must also be considered, as the monolayer may experience a slightly different temperature than is measured by the thermocouple immersed in the subphase liquid. However, we estimate this error to be less than $4^{\circ} \mathrm{C}$ at the lowest temperatures, ${ }^{61}$ which is too small to account for the discrepancy in the inset to Figure 4d. Karim et al. also observed a weakening temperature dependence with decreasing $T$ below $T_{\theta}$, although the effect is less pronounced as their data cover a more limited range of $\tau^{29}$

The magnitude of the contraction observed in the present work can be compared to that reported by Karim et al..$^{29}$ and Auroy et al..$^{28}$ and also to that reported for dilute free chains. ${ }^{49.55-58}$ In the study of Karim et al, for $M=105 \mathrm{~kg} / \mathrm{mol}$ chains $\left(\Sigma=17\right.$ at $\left.T_{\theta}\right)$ the layer height at $\tau=-0.09$ appears to be roughly $60-70 \%$ of that at $T_{\theta}$. For the highest surface densities in Figure $4 a\left(\Sigma=7.6\right.$ and 10.7 at $T_{\theta}$, see Table II), we find $h_{r m s} / h_{r m s} \theta^{\sim}$ $0.65-0.75$ at $\tau=-0.09$. Thus, the magnitude of the contraction observed in the present data is consistent with that observed by Karim et al., considering that a slightly greater contraction is expected in their case due to the higher $\Sigma$. Auroy et al. observed a contraction of the tethered layer to $65 \%$ of that at $T_{\theta}$ at $\tau=-0.15$ for $M=166 \mathrm{~kg} / \mathrm{mol}$ chains $\left(\Sigma=27\right.$ at $\left.\mathrm{T}_{\theta}\right)$. In Figure 4 we find $\mathrm{h}_{\mathrm{ms}} / \mathrm{h}_{\mathrm{ms} \theta} \sim 0.55-0.65$ at $\tau=-0.15$ for the two highest surface densities. Since greater contraction is again expected in their case due to the higher $\Sigma$, there seems to be a small discrepancy. This slight difference may be due to the fact that the tethered chains are composed of different monomers in the two cases. Furthermore, in their case the layer height was valuated ousing a step function

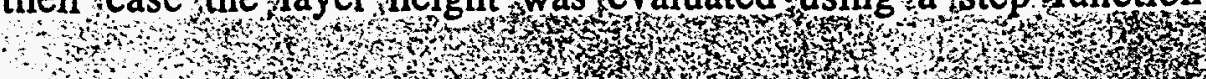
$+4$ 
approximation rather than a detailed profile. Regarding free chains in dilute solution, quite a range of contraction has been reported for different systems, including $R_{g} / R_{g, \theta}=$ 0.67 at $\tau=-.07$ for poly $(\alpha$-methylstyrene) chains with $M=1,400 \mathrm{~kg} / \mathrm{mol}$ in cyclohexane, ${ }^{58} R_{g} / R_{g, \theta}=0.38$ at $\tau=-0.10$ for polystyrene chains with $M=2,000 \mathrm{~kg} / \mathrm{mol}$ in DOP, ${ }^{56} R_{g} / R_{g, \theta}=0.85$ at $\tau=-0.02$ for PS with $M=8,600 \mathrm{~kg} / \mathrm{mol}$ in cyclohexane, ${ }^{49}$ and $R_{g} / R_{g, \theta}=0.80$ at $\tau=-0.03$ for PS with $M=4,600 \mathrm{~kg} / \mathrm{mol}$ in methyl acetate. ${ }^{55}$ Direct comparison of these data to the present data is difficult due to the higher $M$ used in the free chain studies. We also note that the free chain studies were conducted on the low concentration side of the critical point, whereas the present systems are on the high $\sigma$ side of the critical point (discussed below). Nevertheless, a feature in common among all these studies is that at the lowest temperatures the chains are still quite expanded relative to the fully collapsed nonsolvent limit. Due to the very weak temperature dependence in the collapsed regime, it appears to be impractical to reach the nonsolvent limit by cooling below $T_{\theta}$ for systems in which van der Waals interactions dominate. On the other hand, for poly( $\mathrm{N}$-isopropylacrylamide) in water, which involves much stronger hydrogen bonding interactions and possesses a lower critical solution temperature, Wu et al. observed $R_{g} / R_{g . \theta}=0.15$ when this system was heated to just a few degrees above $T_{\theta}{ }^{57}$

In Figure $4 \mathrm{e}$ the data are compared with the scaling relation $h_{r m s} / h_{r m s . \theta} \alpha \tau / \phi_{\theta}$ which is based on an analogy with the swelling of lightly crosslinked gels. ${ }^{50,62}$ Application of this relation supposes strongly interacting tethered systems, but does not require that the tethered layers be in the strongly-stretched asymptotic regime. Following Karim et al ${ }^{30}$ we evaluated $h_{\mathrm{rms}}$ and $h_{\text {dry }}$ directly from the experimental data. Karim et al. observed good agreement with this scaling relationship for several molecular weights ranging from $8.5 \mathrm{~kg} / \mathrm{mol}$ to $105 \mathrm{~kg} / \mathrm{mol}$. Our data do not conform to this universal scaling relationship, as shown in Figure 4e. To understand these widely differing results, it must first be noted that the range of $\Sigma$ for the present data is lower than in the study of Karim et al. The maximum value of $\Sigma$ in the study of Karim et al. $(-17)$ is roughly $50 \%$ 
greater than the maximum value in the present study (10.7). The analogy to a crosslinked gel requires that the tethered layer remain well into the interacting regime over the entire range of $\tau$. This might be the true for their system, considering the higher $\Sigma$ and smaller $\tau$ range, whereas it is not valid for all the systems of the present work. However, we also note that in the work of Karim et al. $\Sigma$ and $M$ were coupled through the grafting process and thus could not be varied independently. In addition, the range of $\tau$ was far more limited than in the present work. Therefore, we believe their comparison does not constitute a rigorous test of this relationship. We note that the curves for the higher $\Sigma$ of the two copolymers in Figure 4e differ most significantly for $\tau<-0.10$, beyond the $\tau$ range in the study of Karim et al.

\section{B. Surface pressure}

\section{Phase behavior}

\section{PDMS homopolymer}

Figure $6 c$ shows that the surface tension of the PDMS monolayer-covered surface increases more rapidly with decreasing $\mathrm{T}$ than that of the bare DOP surface. For low surface concentration, this results in negative pressure at the lowest temperatures. This is most clearly observed at PDMS concentration of $0.7 \mathrm{mg} P D M S / \mathrm{m}^{2}$, as shown in the isotherms in Figure 8b. The origin of a negative surface pressure is the attraction among the PDMS molecules on the surface. For a sufficiently poor solvent, this attraction ultimately leads to a phase separation into polymer-rich and polymer-poor regions, as has been observed for PDMS monolayers on water. ${ }^{63}$. In that case, the surface pressure vanishes at less than monolayer coverage. In the present case, the gradual nature of the decrease in the surface "solvent quality" with decreasing temperature apparently allows 
the PDMS monolayer to remain dispersed at temperatures below the binodal. Baranowski has examined the surface pressure isotherms of PDMS monolayers on a poor solvent by numerical SCF. ${ }^{64}$. Negative surface pressures for PDMS monolayers were obtained at low surface density. In those calculations $\Sigma$ was fixed such the system was not able to phase separate. A detailed study of the entire phase diagram has not yet been performed for this case.

\section{PDMS-PS diblock copolymer}

The negative surface pressures observed for the PDMS-PS block copolymer monolayers in Figure 8b are of greater absolute magnitude than was observed for PDMS monolayers. Attraction among the molecules within the monolayer is again the origin of negative pressure. However with the diblock copolymers, attraction can occur among both the surface blocks and the submerged blocks, which accounts for the greater effect. As in the case of PDMS, slow cooling apparently allows the block copolymers to remain dispersed in the metastable regime (no decrease in reflectivity was observed upon cooling for the series in Figure $8 a$ and $8 b$ ). We have estimated the values of $T$ at which the surface tensions for the copolymer-covered surfaces equal those of the bare DOP surface. These points indicate the binodal in the phase diagram, shown in Figure 9. Clearly more data points are needed to map the entire phase diagram, but the two data points obtained for each copolymer indicate that the present $\sigma$ values are on the high $\sigma$ side of the critical point. Detailed isotherms and phase diagrams have been calculated by Szleifer $e t$ al. using SCMF for both fixed and mobile tethering points. ${ }^{2.15 .16}$ For the latter case they determined a scaled form of the phase diagram, including regions of metastability. The present data for the two copolymers are plotted according to this scaled form in the inset to Figure 9. Precise scaling is not observed. The small discrepancy may be due to contributions of the surface PDMS blocks to the phase behavior. Moreover, contributions from the surface blocks may also account for the fact that the monolayers contributions from the surace bocks may also account for the fact that the mononger 
cross into the metastable regime for $\mathrm{T}$ very close to $\mathrm{T}_{\theta}$ for the submerged PS blocks, rather than for $\mathrm{T}$ well below $\mathrm{T}_{\theta}$ as for free PS chains with molecular weights of $170-330$ $\mathrm{kg} / \mathrm{mol}$ in solution. ${ }^{56}$ The decrease in reflectivity with decreasing $\mathrm{T}$ at low $\sigma$, shown in Figure 7 , is a dramatic indication of the metastable nature of the system. The fact that phase separation at low $\mathrm{T}$ occurs as $\sigma$ is decreased confirms that the present systems are on the high $\sigma$ side of the phase diagram.

\section{Residual pressure at highest $\sigma$ and lowest $T$}

Finally, we retum to an important question motivating this study. Can we infer something about the relative contributions of enthalpic and entropic (chain configuration) effects in the pressure of interacting tethered layers by examining the $T$ dependence of the surface pressure below $T_{\theta}$ ? Here we focus on the higher $\sigma$ monolayers, where positive pressure is observed at all $\mathrm{T}$. The positive pressure is due nearly entirely to the interaction of the submerged PS blocks, since the PDMS blocks are at less then full monolayer coverage (indicated by the relative positions of the isotherms in Figure 8). To account for the small contribution of the PDMS blocks, we define an excess surface pressure $(\Delta \Pi)$ as the difference in pressure for the PDMS-PS and PDMS monolayers at a given PDMS surface concentration. We observe that the large decrease in layer height from $\tau=0$ to $\tau=-0.21$ discussed earlier is accompanied by a surprisingly small decrease in $\Delta \Pi$. This is illustrated in Figure 10, in which the $\Delta \Pi$ isotherms for each copolymer in good and theta solvents obtained previously $y^{39,40}$ are shown along with the present data. At $\sigma=0.73 \times 10^{-4} \AA^{-2}$ for the $28-330$ copolymer, for example, the fractional decrease in layer height from good to theta solvent conditions is comparable to that observed in the present data over the $T$ range from $\tau=0$ to $\tau=-0.21$ at $\sigma=1.2 \times 10^{-4} \AA^{-2}$, ${ }^{65}$ yet the decrease in $\Delta \Pi$ over this $T$ range is much smaller than the difference in $\Delta \Pi$ for good and theta solvent conditions at $\sigma=0.73 \times 10^{-} \AA$, The molecular dynamics simulations of Grest ${ }^{21 b}$ indicate 
a greater shift in the pressure isotherm to higher $\sigma$ from theta to poor solvent conditions, than for good to theta solvent conditions. Therefore, the data in Figure 10 strongly suggest that the surface pressure excess in the present Langmuir monolayer system is pathway dependent (ie. we would expect much lower $\Delta \Pi$ if $\sigma$ were increased to $1.2 \times 10^{-4}$ $\AA^{-2}$ isothermally at $-30 \mathrm{C}$ ).

The reason for such a small decrease in $\Delta \Pi$ accompanying the large decrease in layer height over the present $T$ range is unclear. Previously we have argued that the rapid rise in $\Pi$ with $\sigma$ observed for the highly asymmetric Langmuir diblock copolymer monolayer systems may be due to highly nonGaussian chain configurations in laterally compacted tethered layers. Such distorted configurations will arise if lateral interpenetration is limited by steric effects. We suggest that such distorted configurations only partially relax as the temperature is decreased, accounting for the remaining positive pressure at the lowest $\mathrm{T}$.

\section{SUMMARY}

We have employed Langmuir monolayers of asymmetric polydimethylsiloxanepolystyrene diblock copolymers on dioctyl phthalate at temperatures ranging from $22^{\circ} \mathrm{C}$ to $-35^{\circ} \mathrm{C}$ as a model system for tethered chains in poor solvent conditions. The thickness of the tethered layer decreases continuously with decreasing $T$ for most of the $T$ range, but becomes only weakly dependent on $T$ below $-20 \mathrm{C}$. At the lowest $\mathrm{T}$, the profile is still swollen compared to the fully collapsed, nonsolvent limit. The contraction of the layer is examined as a function of both $\sigma$ and M. For the highest $\sigma$, the monolayers are strongly interacting at $T_{\theta}$ yet they are only weakly interacting at the lowest temperatures. Therefore, the decrease in layer height does not follow the universal scaling predicted for the asymptotic strong-stretching limit, but rather at the lowest $T$ the behavior approaches the universal scaling predicted for noninteracting chains. The best-fit profile contains a depletion layer at the air surface, which increases in magnitude with decreasing $T$. 
The free energy of the layer and the lateral phase behavior is probed through surface tension measurements. The surface tension of DOP with PDMS-PS and PDMS monolayers increases with decreasing $\mathrm{T}$ more rapidly than that of the bare DOP surface. Correspondingly, negative surface pressures are observed at low coverages for both cases, indicating metastability toward lateral phase separation. The effect is more pronounced for the block copolymers since attractive interactions can occur among the PDMS blocks on the surface as well as among the PS blocks below the surface. At low PDMS-PS coverages, a dispersed phase/condensed phase transition with decreasing $T$ was indicated by a dramatic decrease in reflectivity. The fact that a phase boundary is crossed with decreasing $\sigma$ indicates that the present systems are on the high $\sigma$ side of the critical point. At high coverage, only a modest decrease in surface pressure is observed over the present $\mathrm{T}$ range, in spite of the large decrease in layer height. From this result we infer that the pressure in the present Langmuir monolayer system is pathway dependent, and thus that nonequilibrium effects (such as distorted chain configurations) likely account for a large proportion of the elevated pressures observed in these systems.

ACKNOWLEDGMENTS We are indebted to J. Douglas for a critical reading of this manuscript and providing many insightful comments. This work was partially supported by the U.S. Department of Energy under contracts DE-AC04-94AL85000, W-7405ENG-36, and W-31-109-ENG-38 and benefited from the use of the NG7 reflectometer at the National Institute of Standards and Technology. 


\section{REFERENCES AND NOTES}

[1] A. Halperin, M. Tirrell, and T.P. Lodge, Adv. Polm. Sci., 100, 31, (1992).

[2] Szleifier, I Adv. Chem. Phys., 94, 165, (1996).

[3] G.S. Grest and M. Murat, Monte Carlo and Molecular Dynamics Simulations in Polymer Science; Binder, K., Ed.; Clarendon Press: Oxford, 1994.

[4] S. T. Milner, Science 251, 905, (1991).

[5] E. Raphael and P. G. de Gennes, J. Phys. Chem. 96, 4002, (1992); H. Ji, and P. G. de Gennes Macromolecules 26, 520, (1993).

[6] M. Amiji and K. Park. J. Biomater. Sci. Polym. Ed. 4. 217, (1993).

[7] D. H. Napper, Polymeric Stabilization of Colloid Dispersions, Academic Press, New York, 1983.

[8] A. Halperin, J. Phys. (Paris) 49, 547, (1988).

[9] D. F. K. Shim and M. E Cates, J. Phys. (Paris) 50, 3535, (1989).

[10] E. B. Zhulina, O. V. Borisov, V. A. Pryamitsyn, and T. M. Birshtein, Macromolecules 24, 140, (1991).

[11] M. Muthukumar and J.S. Ho, Macromolecules 22, 965, (1989). 
[12] M. D. Whitmore and J. Noolandi, Macromolecules 23, 3321, (1990).

[13] K. Huang and A. Balazs, Macromolecules, 26, 4736, (1993).

[14] C. Yeung, A. C. Balazs, and D. Jasnow, Macromolecules 26, 1914, (1993).

[15] M. A. Carignano and I. Szleifier, J. Chem. Phys. 100, 3210, (1994).

[16] H. Tang, M. A. Carignano, and I. Szleifier, J. Chem. Phys. 102, 3404, (1995).

[17] P. Y. Lai and K. Binder, J. Chem. Phys. 95, 9288, (1991).

[18] P. Y. Lai, Comp. Polym. Sci. 2, 157, (1992).

[19] J. D. Weinhold and S. K. Kumar, J. Chem. Phys. 101, 4312, (1994).

[20] K. G. Soga, H. Guo, and M. J Zuckermann, Europhys. Lett., 29, 531, (1995).

[21] G. S. Grest and M. Murat, 26, 3108, (1993); G. S. Grest, Macromolecules 27, 418, (1994).

[22] E. A. Di Marzio, Macromolecules 17, 969, (1984).

[23] I. C. Sanchez, Macromolecules 12, 980, (1979).

[24] C. Williams, F. Brochard, and H.Firsch, Ann. Rev. Phys. Chem., 32, 433, (1981). 
[25] D. R. M. Williams, J. Phys. II, 3, 1313, (1993).

[26] D. Perahia, D. G. Wiesler, S. K. Satija, L. J. Fetters, S. K. Sinha, and S. L. Milner, Phys. Rev. Lett. 72, 100, (1994); D. Perahia, D. G. Wiesler, S. K. Satija, L. J. Fetters, S. K. Sinha, and G. S. Grest, Physica B 221, 337, (1996).

[27] P. Auroy, L. Auvray, and L. Leger Macromolecules 24, 2523, (1991).

[28] P. Auroy, L. Auvray, and L. Leger Macromolecules 25, 4134, (1992).

[29] A. Karim, S. K. Satija, J. F. Douglas, J. F. Ankner, and L. J. Fetters, Phys. Rev. Lett. 73, 3407, (1994).

[30] A. Karim. J. F. Douglas. F. Horkay, L. J. Fetters, and S. K. Satija, Physica B 221 , 331. (1996).

[31] P. Gallagher and S. Satija, in Proceedings of the 5th Surface X-ray and Neutron Scattering Conference, Oxford, July 1997, edited by P. Gallagher and S. Satija, Proceedings of the International Conference on Neutron Scattering, Toronto, August 1997.

[32] S. M. Baker, N. Melosh, E. Torgerson, G. Smith, C. Toprakcioglu, and A. Vradis, (in preparation).

[33] S. J. O'Shea, M. ER. Welland, and T. Rayment, Langmuir, 9, 1826, (1993). 
[34] W. Zhao, G. Krausch, M. H. Rafailovich, and J. Sokolov, Macromolecules, 27, 2933, (1994).

[35] A. Karim, V. V. Tsukruk, J. F. Douglas, S. K. Satija, L. J. Fetters, D. H. Reneker, and M. D. Foster, J. Phys. II, 5, 1441, (1995).

[36] S. M. Baker, A. Callahan, G. Smith, C. Toprakcioglu, A. Vradis, in preparation

[37] M.S. Kent, L.T. Lee, B. Farnoux, and F. Rondelez, Macromolecules 22, 1449, (1989).

[38] B. J. Factor, L. T. Lee, M. S. Kent, and F. Rondelez, Phys. Rev. E. 48, 2354, (1993).

[39] M.S. Kent, L.T. Lee, B.J. Factor, F. Rondelez, and G. Smith. J. Chem. Phys. 103(6), $2320,(1995)$.

[40] M.S. Kent, J. Majewski, G. Smith, L.T. Lee, S. Satija, J. Chem. Phys. 108(13), $5635,(1998)$.

[41] L.T. Lee, B.J. Factor, M.S. Kent, and F. Rondelez J. Chem. Soc., Faraday Discuss. 98, 139, (1994).

[42] L. T. Lee and M. S. Kent Phys. Rev. Lett, 13, 2899, (1997).

[43] F. E. Runge, M. S. Kent, and H. Yu, Langmuir 10, 1962, (1994). 
[44] M. S. Kent, B. J. Factor, S. Satija, P. Gallagher, and G. S. Smith, Macromolecules 29, 2843, (1996).

[45] G. C. Berry, J. Chem. Phys., 44, 4550, (1966).

[46] H. Ahrens, S. Forster, and C. A. Helm, Macromolecules 30, 8447, (1997).

[47] Use of a commercial product does not imply recommendation or endorsement by NIST, nor does it imply that the product is necessarily the best available.

[48] N. P. Balsara, D. Perahia, C. R. Safinya, M. Tirrell, and T. P. Lodge, Macromolecules 25, 3896, (1992).

[49] I. H. Park, Q. W. Wang, and B. Chu, Macromolecules 20, 1965, (1987).

[50] M. Zrinyi and F. Horkay, Macromolecules 22. 394. (1989).

[51] For a surface with heterogeneity on a scale which is large compared with the coherence length of the neutron beam, the reflectivity is an area-weighted average of the intensity reflected from each region. In this case, the area fraction occupied by the islands of copolymer cannot be determined precisely because the reflectivity from the islands is not known. However, an upper bound of $20 \%$ is obtained using the reflectivity from the dispersed state in the inset to Figure 7. The dimension normal to the surface is nearly the same in the islands as in the dispersed state, as can be seen from the comparable peak positions for the data at $-30 \mathrm{C}$ in Figure 7 and in the inset. 
[52] S. Wu, Polymer Interface and Adhesion, (MARCEL DEKKER, Inc.) New York, 1982.

[53] R. Baranowski and M. D. Whitmore, J. Chem. Phys. 103(6), 2340, (1995).

[54] R. Baranowski and M. D. Whitmore, J. Chem. Phys. 108, 9885, (1998).

[55] B. Chu, I. H. Park, Q. W. Wang, and C. Wu, Macromolecules 20, 2833, (1987).

[56] P. Stepanek, C. Konak, and B. Sedlacek, Macromolecules 15, 1214, 1992.

[57] C. Wu and S. Zhou, Macromolecules 28, 8381, (1995).

[58] J. Li, S. Harville, and J. W. Mays, Macromolecules 30, 466, (1997).

[59] Since $R_{z}$ for a free PS chain in dilute solution is not available at the lower temperatures of this study, we estimated the value of $\Sigma$ at $-30^{\circ} \mathrm{C}$ using the contraction of

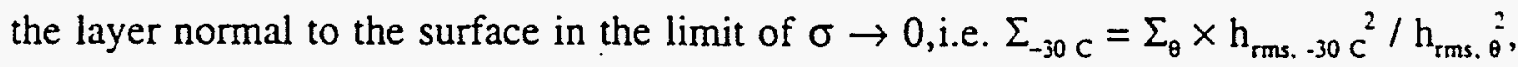
where $h_{\text {rms. }-30 \mathrm{C}} / h_{\text {rms, } \theta}$ in the limit of $\sigma \rightarrow 0$ was evaluated from the data for the lowest $\sigma$ for each copolymer in Figure 4d. Our previous work showed that $h_{\mathrm{rms}, g o o d} / h_{\mathrm{rms}, \mathrm{g}}$ in the limit of $\sigma \rightarrow$ Us an excellent approximation to $R_{g, g o d} / R_{g, \theta}$ (Ref. 40), and here we assume that the same holds for the ratios in theta and poor solvents.

[60] Our previous work has shown that the $h_{m s} / R_{g}$ is a linear function of $\Sigma$ over this range (Ref. 40). 
[61] This upper limit to the magnitude of the temperature gradient through the liquid was determined by varying the vertical position of the thermocouple, which resulted in a variation of 3.4 degrees at the lowest temperature. This is an upper limit since the thermocouple may have been partially exposed to the air above the liquid at its highest vertical position. A different method with finer spatial resolution is required to precisely determine the gradient. In addition to the above, the fact that the surface tension continues to increase nearly linearly down to the lowest temperatures (see Figure 6) indicates that the surface layer is experiencing nearly the same variation in temperature as measured by the thermocouple down to the lowest temperatures. From the linearity in the data for DOP, the temperature gradient would appear to be less than $4^{\circ} \mathrm{C}$.

[62] This relationship is equivalent to the analytical SCF result derived by Zhulina et al. if $h_{\mathrm{ms} . \theta}$ and $h_{\mathrm{dry}}$ are evaluated in the strong stretching limit.

[63] E. K. Mann. S. Henon. D. Langevin. and J. Meunier. J. Phys. II. 2. 1683. 1992.

[64] R. Baranowski. Ph. D. Thesis, Dept. of Physics, Memorial University of Newfoundland, (1997).

[65] The ratio of the layer height in good and theta solvents is 0.55 for the $28-330$ copolymer at $\sigma=7.3 \times 10^{-5}$ chains $/ \AA^{2}$, and 0.59 for the $20-170$ copolymer at $\sigma=1.5$ $\times 10^{-4}$ chains $/ \AA^{2}$. These values are comparable to the $h_{\mathrm{rms}} / h_{\mathrm{ms}, \theta}$ values at $\tau=-0.21$ in Figure 4a. 
Table I. Surface densities obtained from integrating the profiles

\begin{tabular}{lccc} 
copolymer & $\mathrm{T}\left({ }^{\circ} \mathrm{C}\right)$ & integral $(\AA)$ & \multicolumn{2}{c}{ surface density $\left(10^{-4}\right.$ chains $\left./ \AA^{2}\right)$} \\
\cline { 2 - 4 } $20-170$ & 22 & 41.6 & 1.67 \\
& -1 & 43.3 & 1.74 \\
& -10 & 40.9 & 1.66 \\
& -15 & 41.2 & 1.78 \\
& -25 & 43.2 & 1.74 \\
$20-170$ & -34 & 44.3 & 1.65 \\
& & & \\
& 22 & 14.6 & 0.588 \\
& -2 & 14.2 & 0.572 \\
& -15 & 14.3 & 0.576 \\
& -20 & 14.4 & 0.580 \\
& -25 & 13.7 & 0.551 \\
& -30 & 14.6 & 0.588 \\
& & & \\
& 22 & 56.6 & 1.12 \\
& 0 & 58.6 & 1.21 \\
& -10 & 59.9 & 1.23 \\
& -20 & 61.6 & 1.27 \\
& -30 & 62.2 & 1.28 \\
& -35 & 60.1 & 1.24 \\
& 22 & & \\
& 22 & 25.2 & 0.519 \\
& 0 & 24.5 & 0.505 \\
& -20 & 24.7 & 0.540
\end{tabular}


Table I. $\Sigma$ values indicating degree of chain overlap

$\begin{array}{lcc}\text { copolymer } & \underline{\Sigma} \text { at } 22{ }^{\circ} \mathrm{C}(\tau=0) & \underline{\Sigma} \text { at }-30^{\circ} \mathrm{C}(\tau=-0.21)^{59} \\ 20-170 & 2.6 & 1.3 \\ 20-170 & 7.6 & 3.8 \\ 28-330 & 4.5 & 1.9 \\ 28-330 & 10.7 & 4.4\end{array}$




\section{Figure Captions}

1. Schematic diagram of the temperature-controlled Langmuir trough used in the neutron reflection experiments.

2. Reflectivity data over temperatures ranging from $22^{\circ} \mathrm{C}\left(\mathrm{T}_{\theta}\right)$ to $-35^{\circ} \mathrm{C}$ for a) $20-170, \sigma$ $\cong 1.7 \times 10^{-4} \AA^{-2}$, b) $20-170, \sigma \cong 5.8 \times 10^{-5} \AA^{-2}$, c) $28-330, \sigma \cong 1.2 \times 10^{-4} \AA^{-2}$, d) $28-330, \sigma$ $\cong 5.2 \times 10^{-5} \AA^{-2}$. Curves for different temperatures have been shifted on the vertical axis for clarity. Actual temperatures are given in Table I. e) Reflectivity from the bare DOP surface at $22^{\circ} \mathrm{C}(\bullet)$ and at $-30^{\circ} \mathrm{C}(+)$.

3. Best-fit profiles corresponding to the curves through the data in Figure 2 for: a) 20 $170, \sigma \cong 1.7 \times 10^{-4} \AA^{-2}$, b) $20-170, \sigma \equiv 5.8 \times 10^{-5} \AA^{-2}$, c) $28-330, \sigma \cong 1.2 \times 10^{-4} \AA^{-2}$, d) $28-$ $330, \sigma \cong 5.2 \times 10^{-5} \AA^{-2}$.

4. Variation of a) rms layer height $h_{r m s}$ and b) maximum volume fraction $\mathrm{O}_{\mathrm{PS} \text {. max }}$ of the segmental concentration profile with reduced temperature $\tau$ for the 20-170 copolymer (open symbols) and the 28-330 copolymer (filled symbols). In each case, circles (squares) represent lower (higher) surface densities. The profile becomes weakly dependent on $\mathrm{T}$ below $-20^{\circ} \mathrm{C}$. In b), the error bars are comparable to the size of the symbols. c) Data for $h_{\text {mms }}$ plotted according to the scaling prediction of the SCF theory of Zhulina et al. ${ }^{10}$ for the asymptotic limit of strongly stretched chains $(\Sigma \gg 1)$. The symbols have the same meaning as in a). d) Data for $h_{\mathrm{rms}}$ plotted according to the scaling prediction in the limit of isolated chains $(\Sigma<1)$. The symbols have the same meaning as in a). The strong dependence on $\Sigma$ at $T_{\theta}$ is due to the stretching of chains within the interacting tethered layers. The dependence on $\Sigma$ decreases with decreasing $T$. At the lowest temperatures, the data approach the universal scaling predicted for the isolated 
chain limit. However, the inset shows that the dependence on temperature is weaker than $h_{m s} \alpha||^{-1 / 3}$ predicted for isolated free chains in the collapsed regime. e) Data for $h_{\text {rus }}$ plotted according to a scaling prediction derived by analogy with the swelling of lightly crosslinked gels. ${ }^{30,50}$ The symbols have the same meaning as in a).

5. Comparison of the best-fit profiles in the low temperature limit for the $20-170$ copolymer at $\sigma$ of $1.7 \times 10^{-4} \AA^{-2}\left(\mathrm{~T}=-35^{\circ} \mathrm{C}\right)$ and $5.8 \times 10^{-5} \AA^{-2}$, (T=-30 $\left.{ }^{\circ} \mathrm{C}\right)$.

6. Variation of surface tension with temperature for a) 20-170 on DOP at $\sigma$ of $5.8 \times 10^{-5}$ $\AA^{-2}(\bullet)$ and $1.7 \times 10^{-4} \AA^{-2}(\boldsymbol{\square})$, b) 28-330 on DOP at $\sigma$ of $5.2 \times 10^{-5} \AA^{-2}(\bullet)$ and $1.2 \times 10^{-4}$ $\AA^{-2}(\mathbb{\square})$, and c) PDMS on DOP at $\sigma$ of $5.0 \times 10^{-5} \AA^{-2}(\bullet), 1.3 \times 10^{-4} \AA^{-2}(+), 2.0 \times 10^{-4} \AA^{-2}$ (४), $2.8 \times 10^{-4} \AA^{-2}(\nabla), 3.8 \times 10^{-4} \AA^{-2}(\mathbf{\Delta}), 7.6 \times 10^{-4} \AA^{-2}(\boldsymbol{\square})$. In c), the error bars are comparable to the size of the symbols. Data are also shown for the bare DOP surface () for comparison.

7. Reflectivity for a $20-170$ copolymer monolayer at $\sigma$ of $4.9 \times 10^{-5} \AA^{-2}$ at $22{ }^{\circ} \mathrm{C}(\bullet)$ and $-30^{\circ} \mathrm{C}(\square)$. Reflectivity at $-30^{\circ} \mathrm{C}$ is significantly decreased relative to that at $22^{\circ} \mathrm{C}$. This decrease in reflectivity in interpreted as indicating a lateral phase transition. Inset shows reflectivity for the same copolymer at a slightly higher $\sigma$ of $5.8 \times 10^{-5} \AA^{-2}$ at $22^{\circ} \mathrm{C}(\bullet)$ and $-30^{\circ} \mathrm{C}(\square)$. For this slightly higher $\sigma$, where no lateral phase transition occurs, there is a large increase in reflectivity over this $\mathrm{T}$ range.

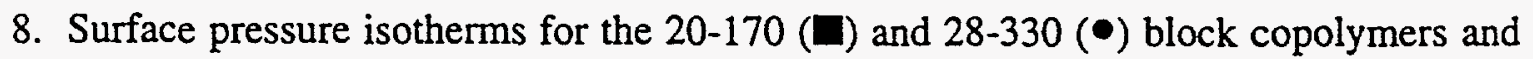
PDMS homopolymer (solid line) on DOP at a) $22^{\circ} \mathrm{C}$ and b) $-30^{\circ} \mathrm{C}$.

9. Phase diagram for the block copolymers on DOP. The data points indicate zero surface pressure (binodal) for the 20-170 ( $\square$ ) and 28-330( $\bullet$ ) copolymers. For the higher 
surface densities, the temperature at which the pressure vanishes was determined by extrapolating the data in Figure 6. The inset compares the data to the scaling form predicted by Szleifer et $a l^{2,15,16}$ The lack of precise scaling may be due to contributions of the surface PDMS blocks to the phase behavior.

10. Decrease in excess surface pressure $(\Delta \Pi)$ observed over the temperature range from $\tau=0$ (田) to $\tau=-0.21$ (口) compared to the $\Delta \Pi$ isotherms measured previously in good $(\mathrm{O})^{39}$ and theta $(\bullet)^{40}$ solvents for a) the 20-170 copolymer and b) the 28-330 copolymer. The decrease in $\Delta \Pi$ over the present $T$ range is smaller than expected based on the difference in the isotherms in good and theta solvent conditions. This seems to suggest a non-equilibrium or pathway dependent effect. 


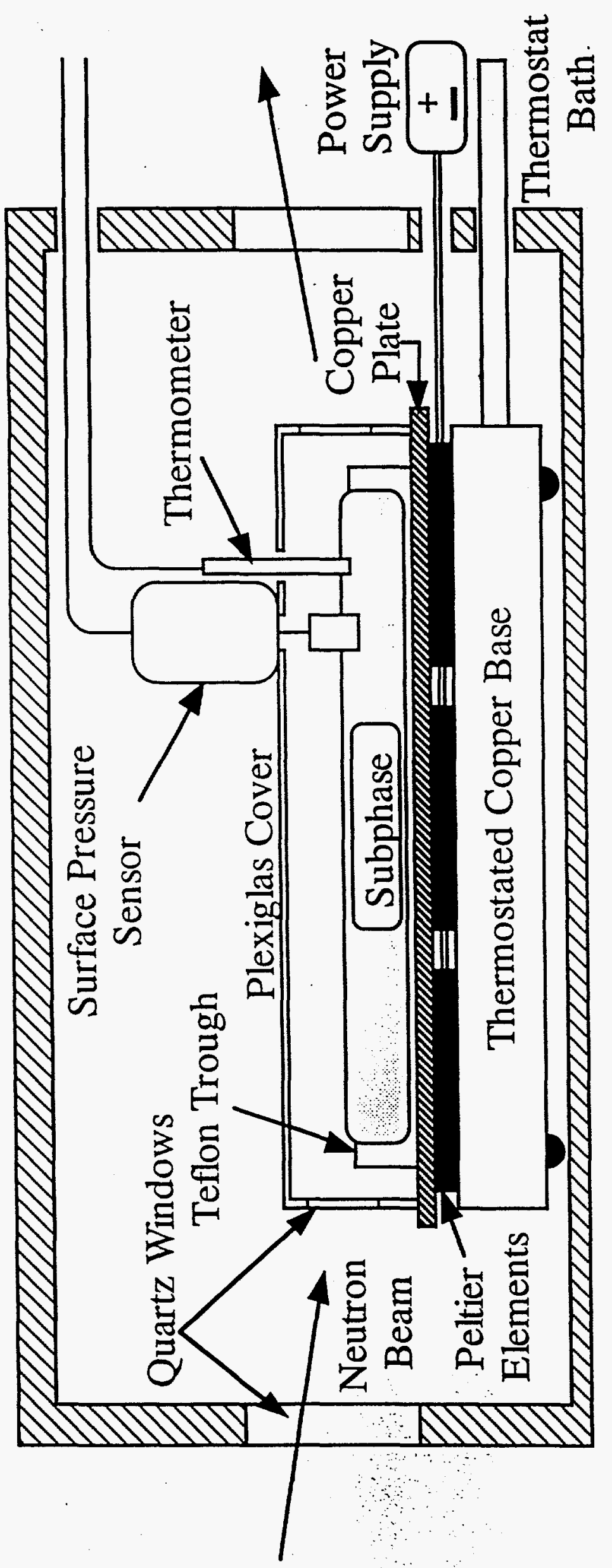




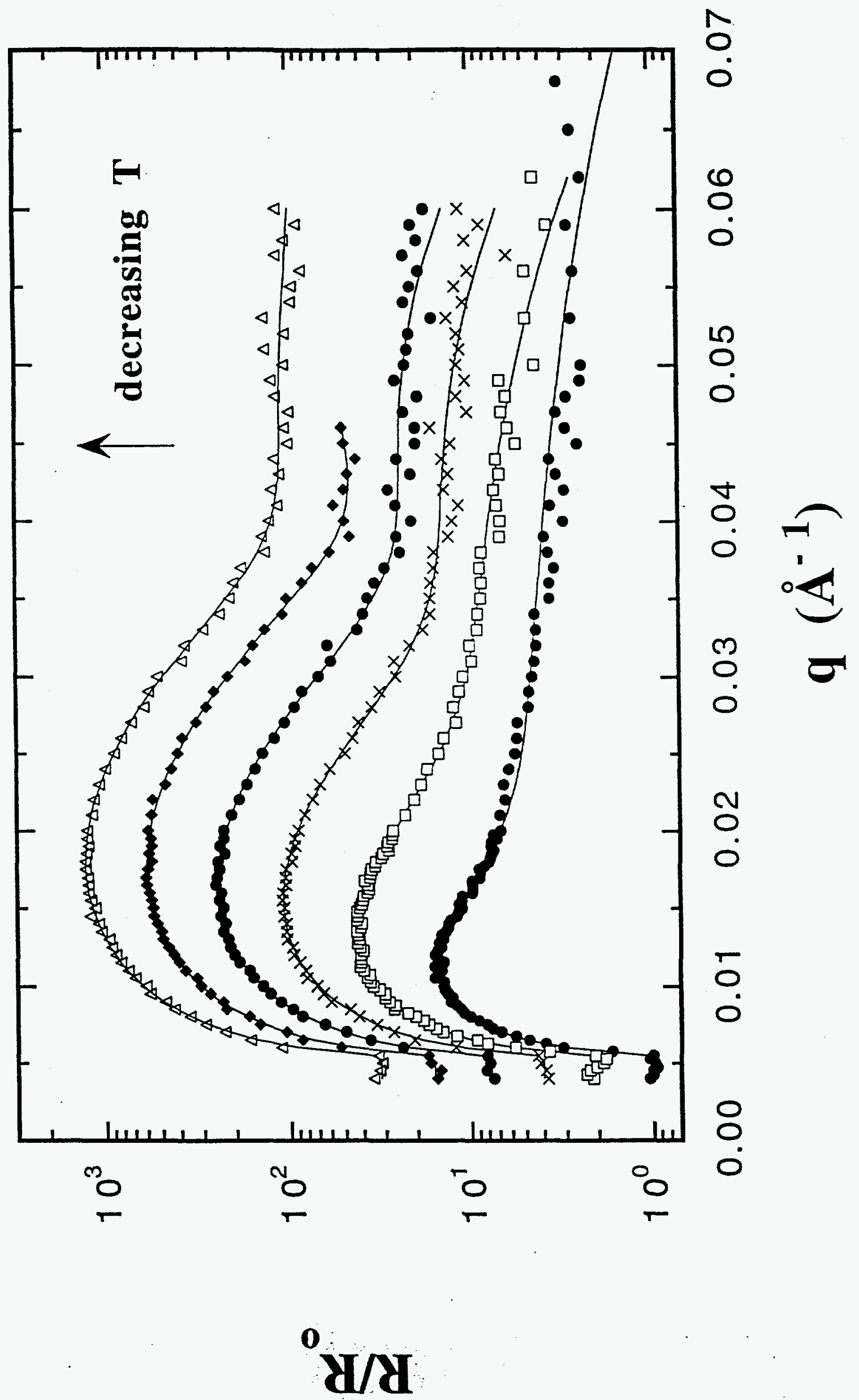




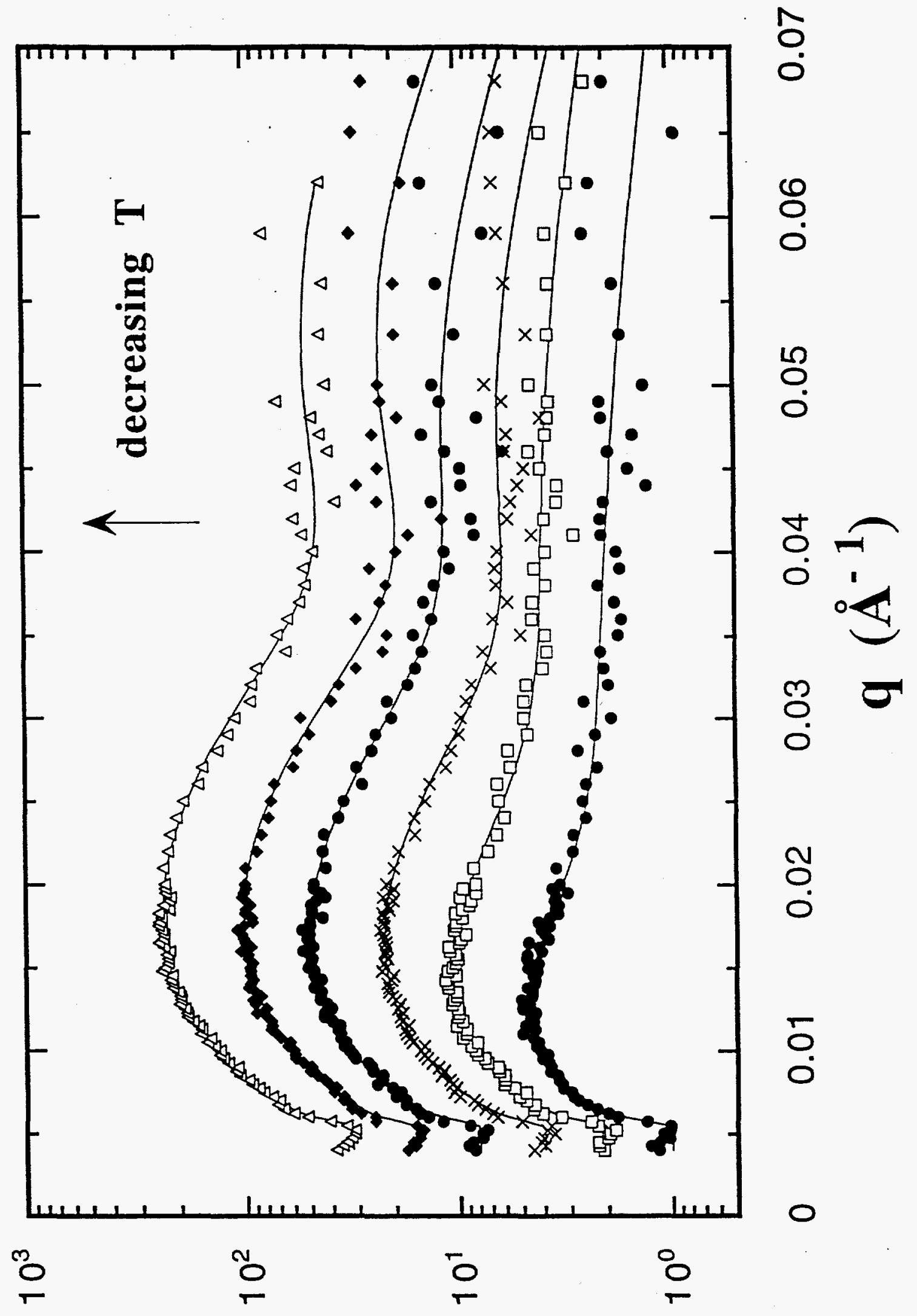

0 


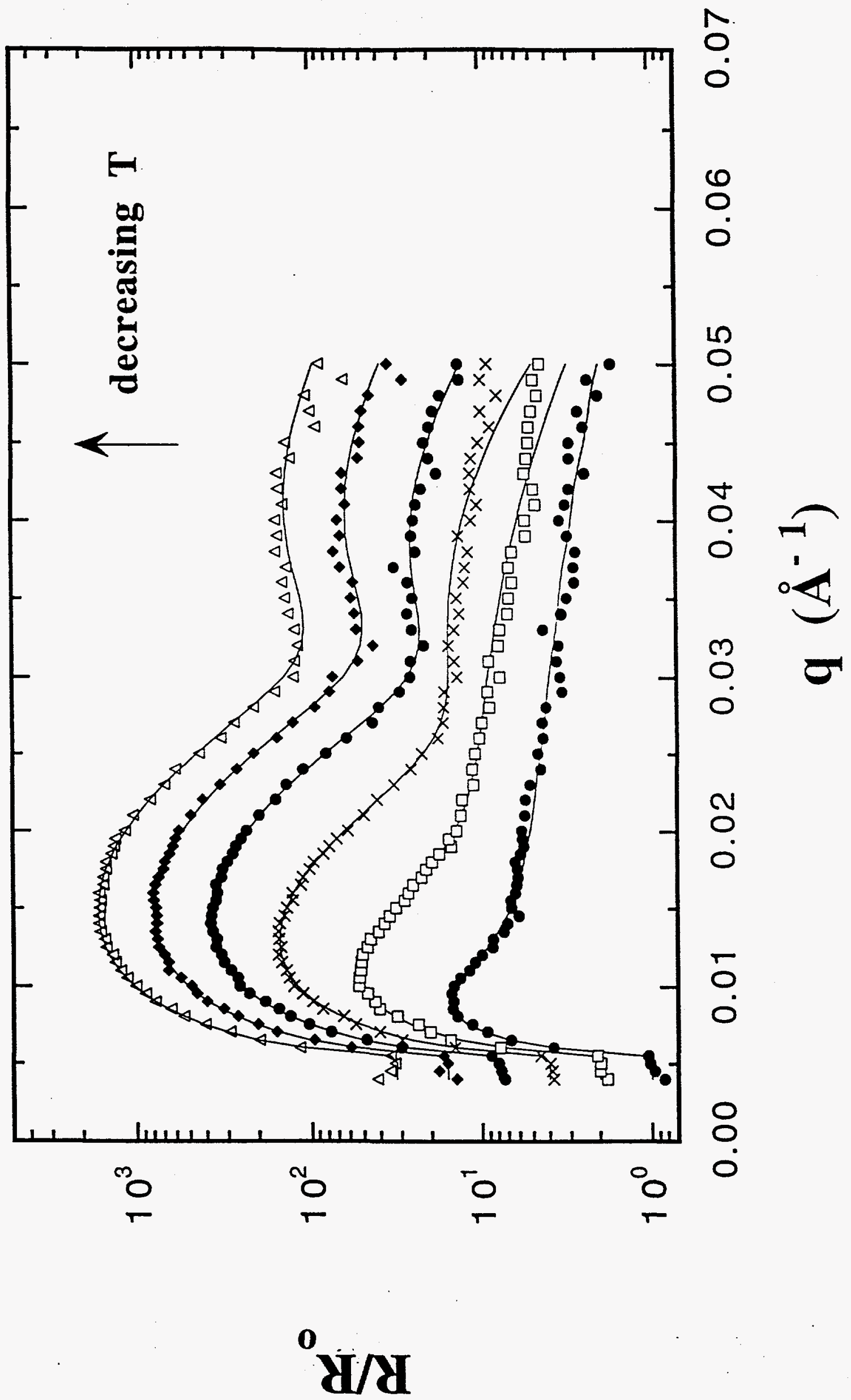




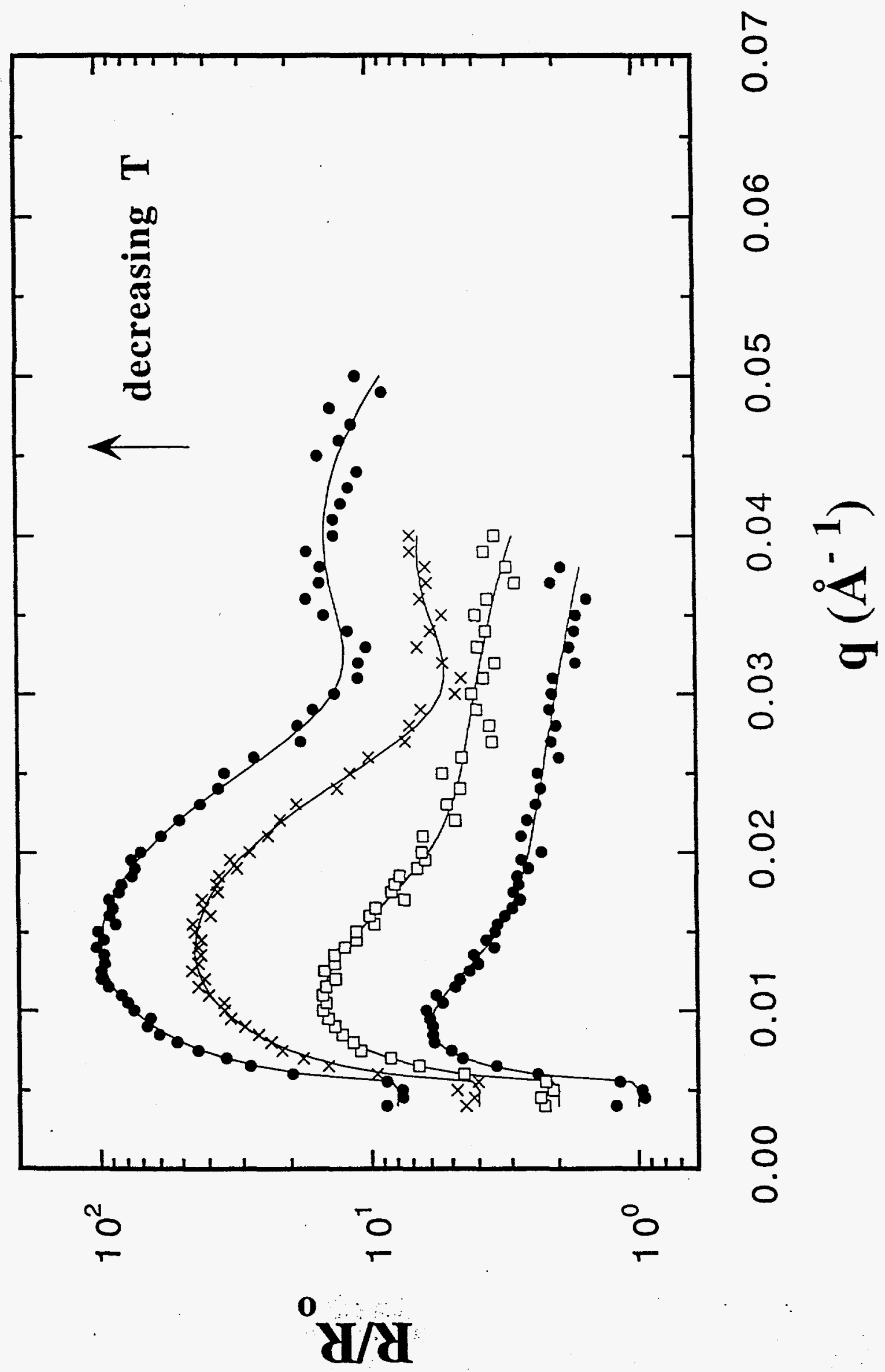




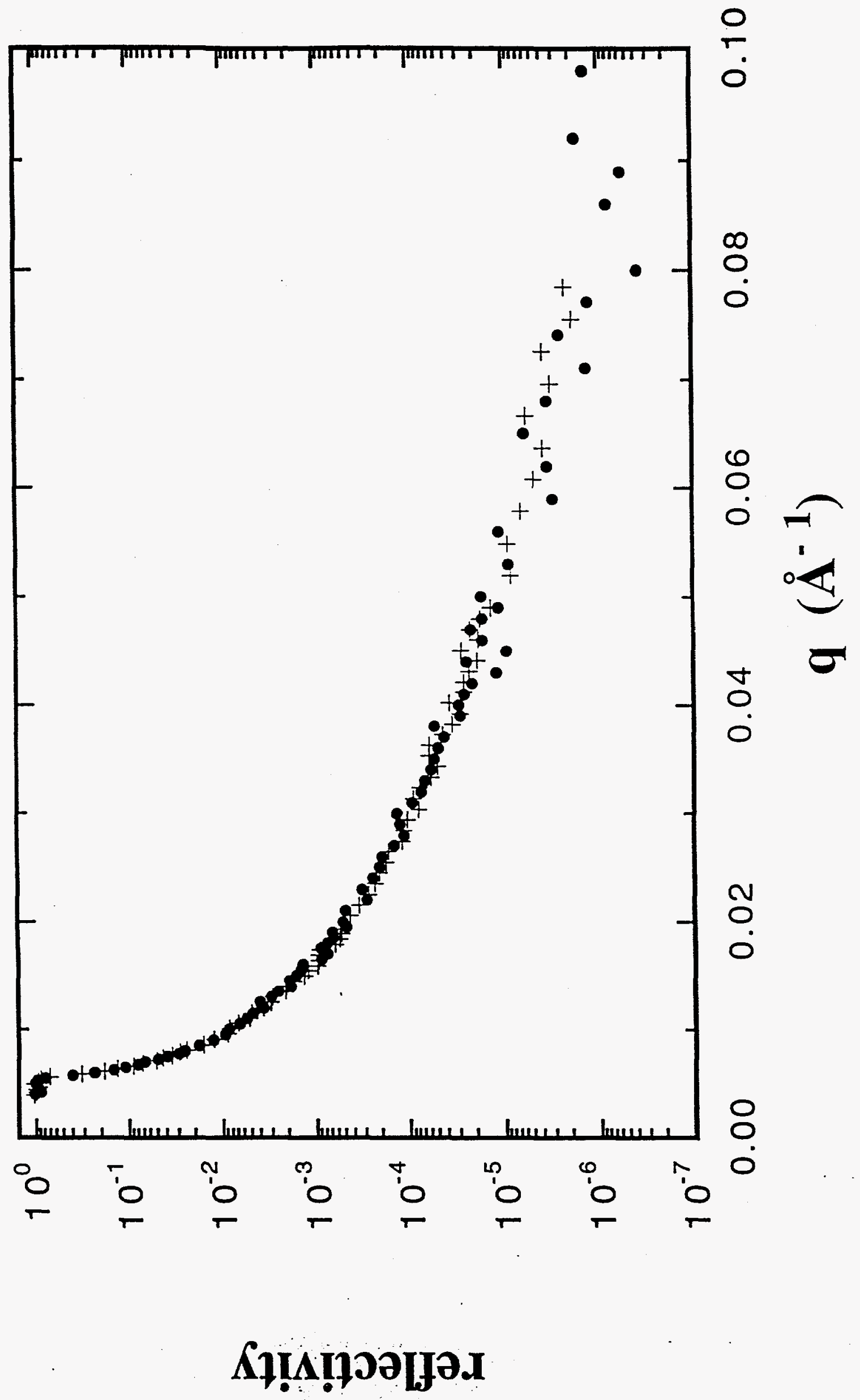




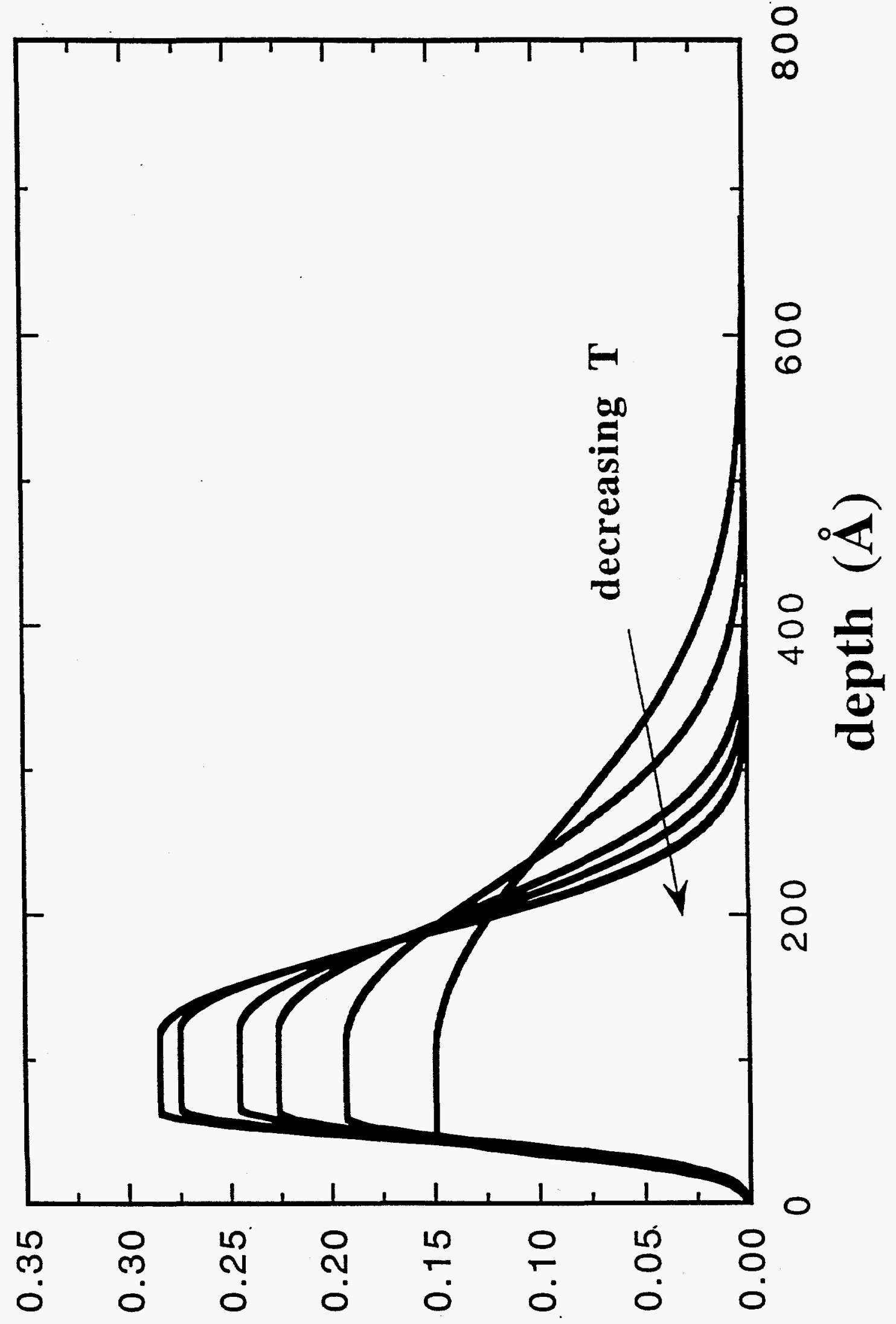

Sd p uọpe.dy aunIoג 


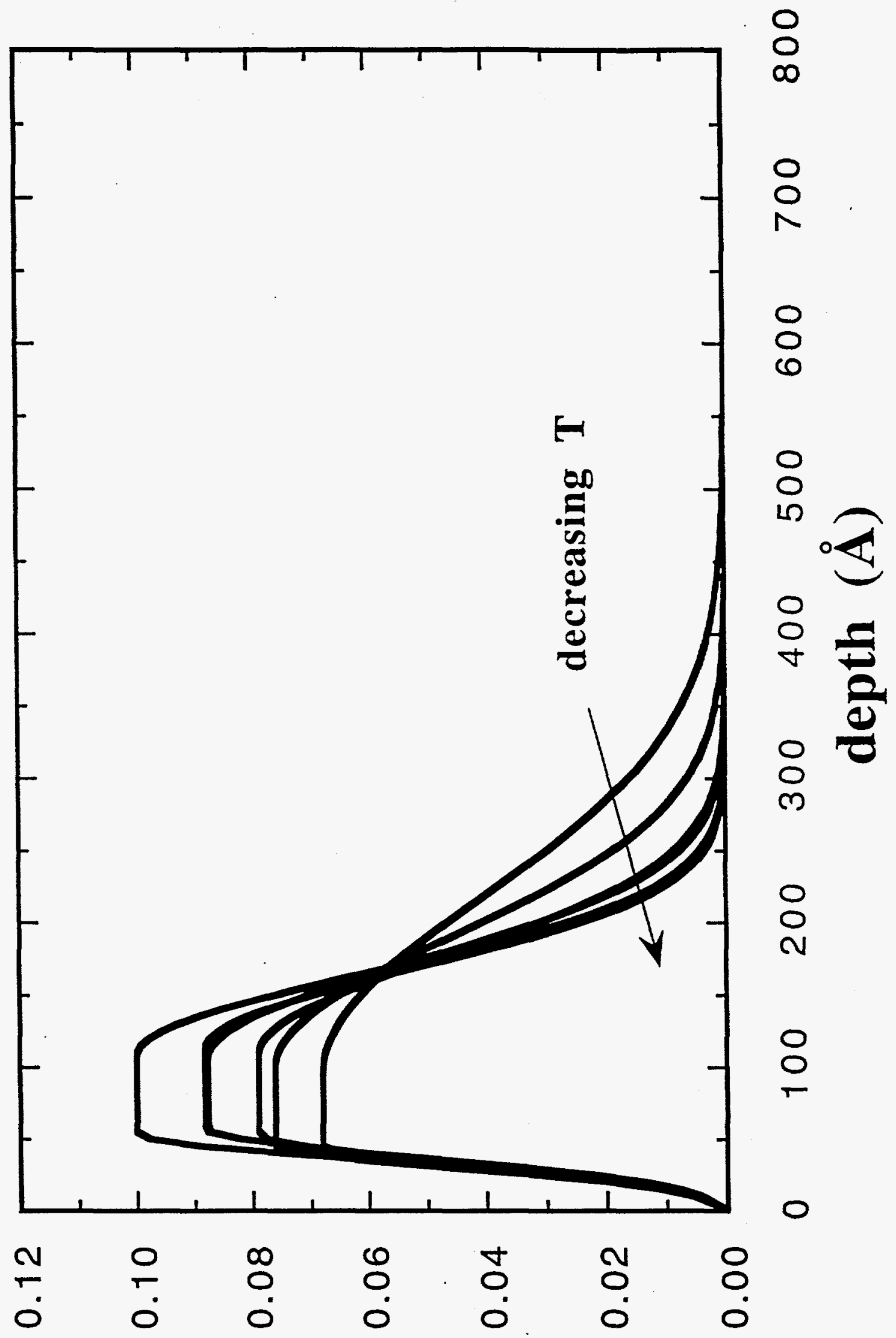

Sd-p uọpedy oun!os 


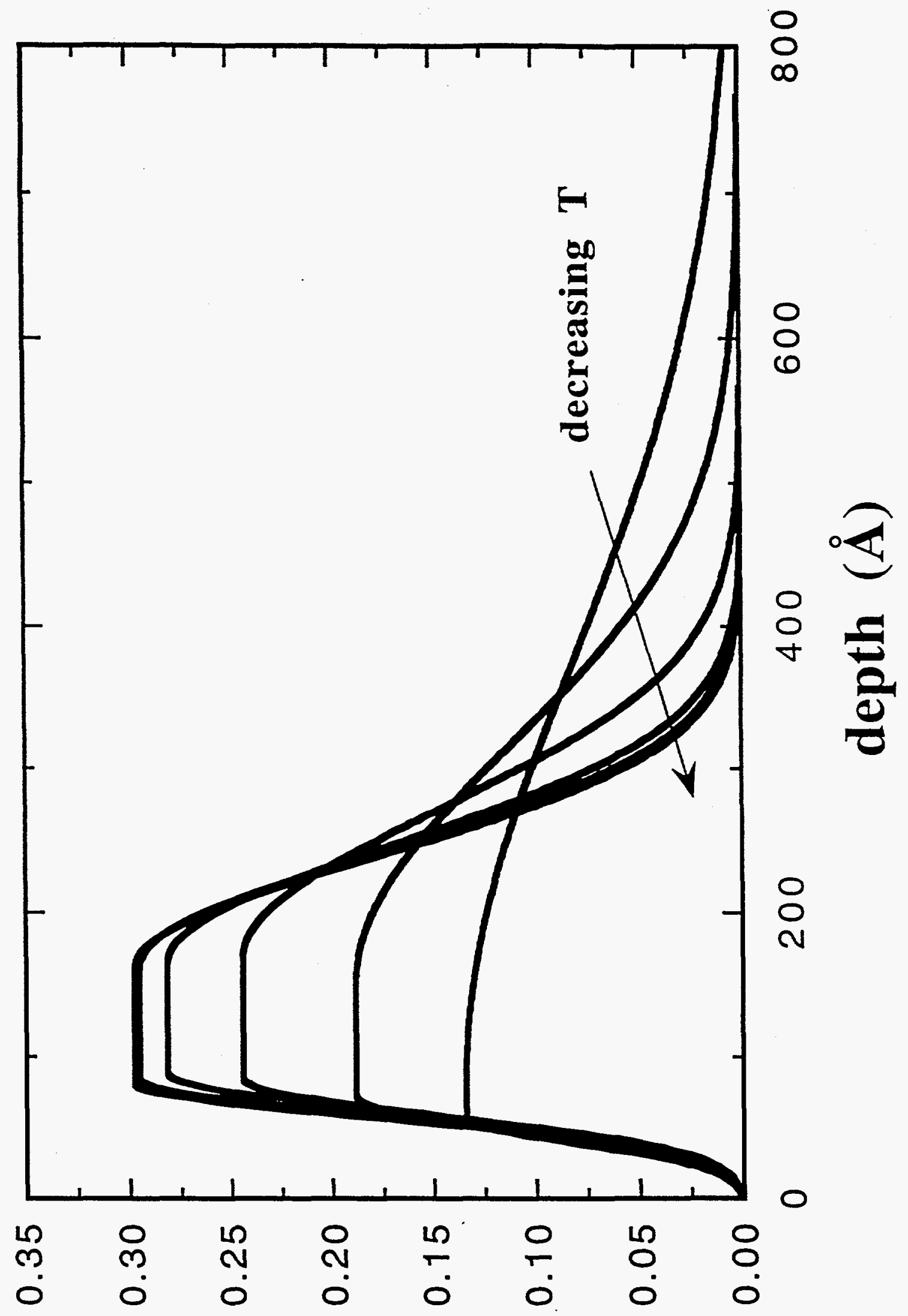

Sd-p uọpedy oum[on 


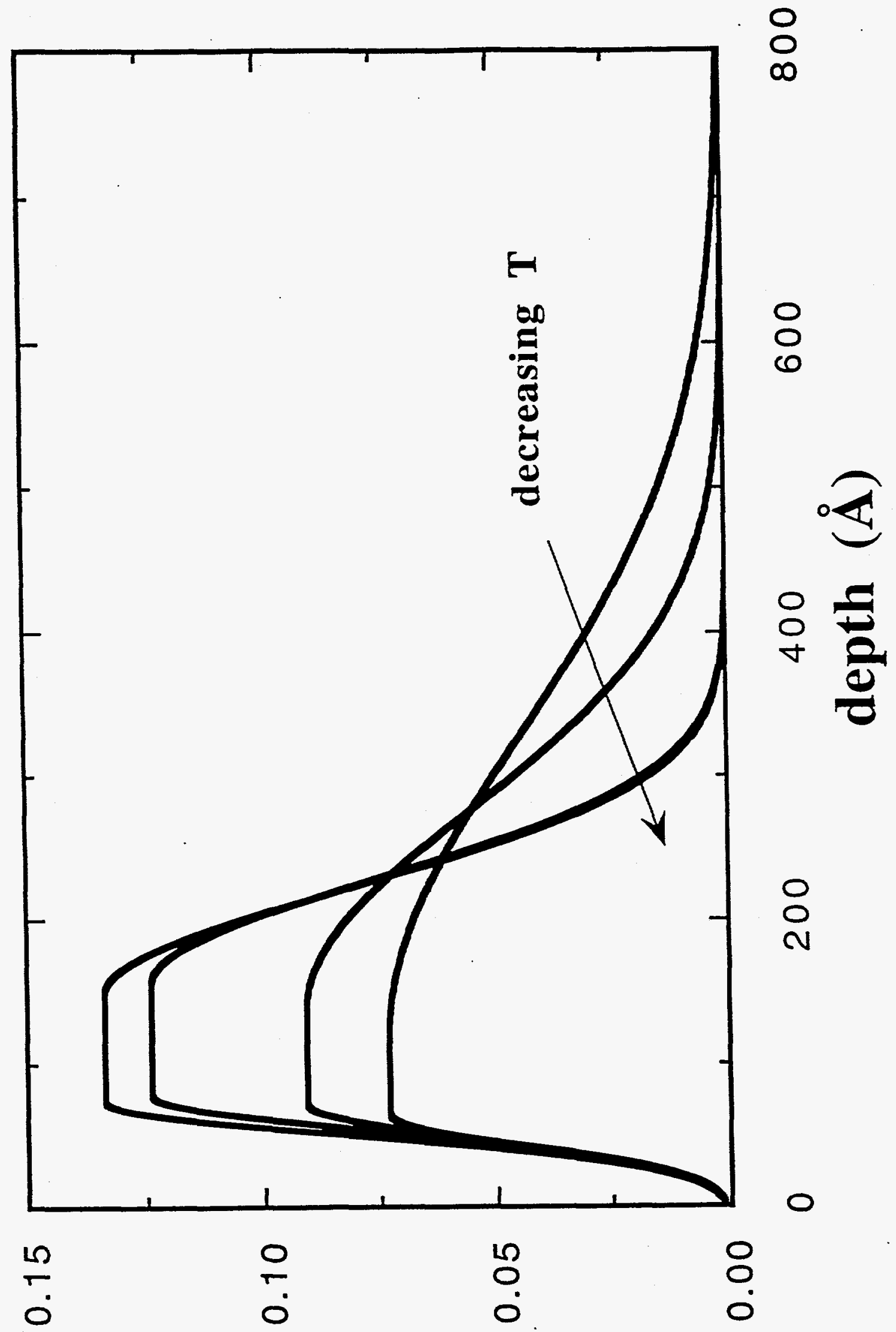

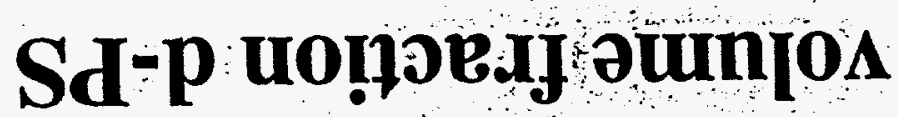




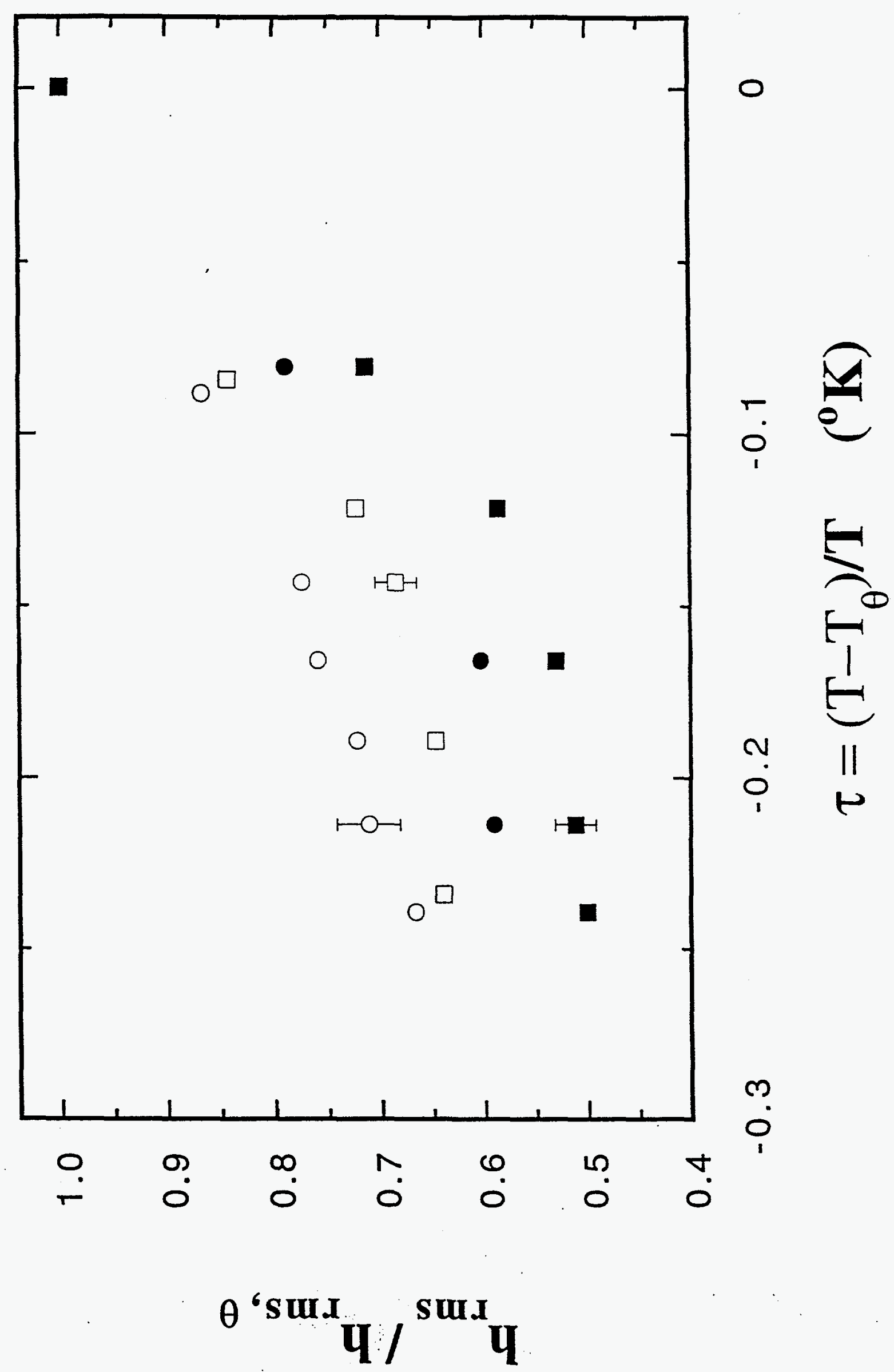




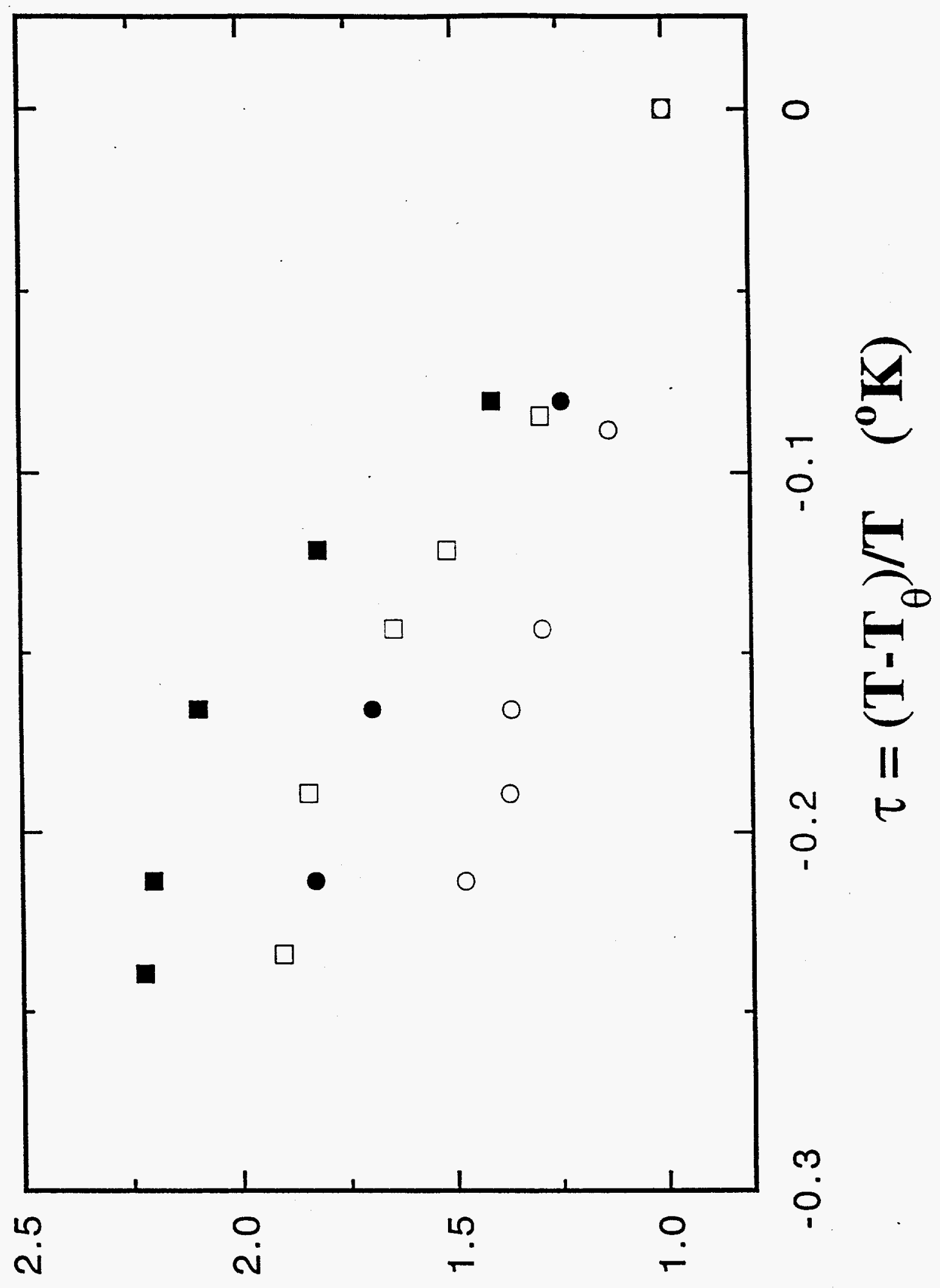

$\theta^{6} \operatorname{xeu}_{\phi} /$ xeu $_{\phi}$ 


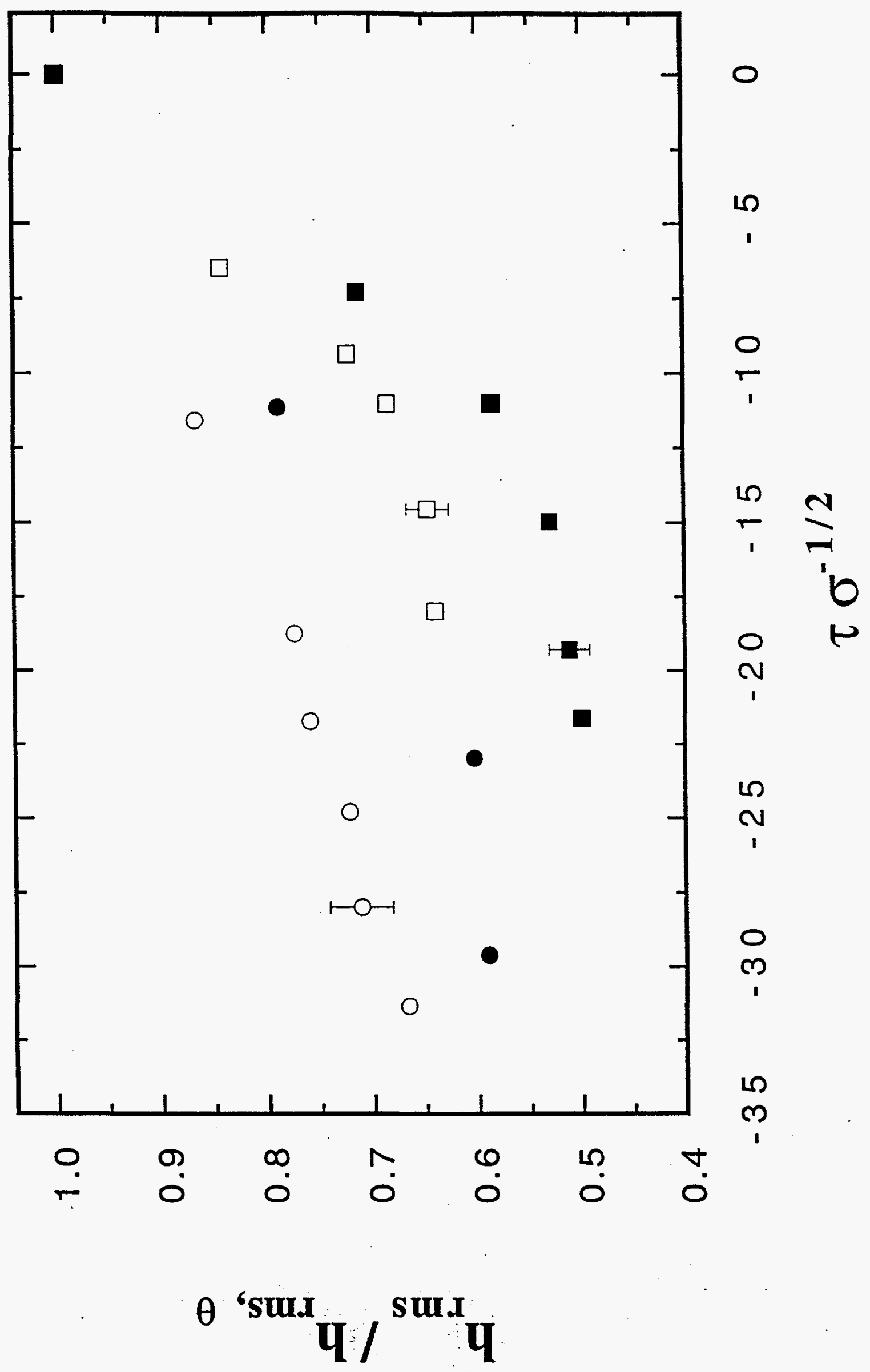




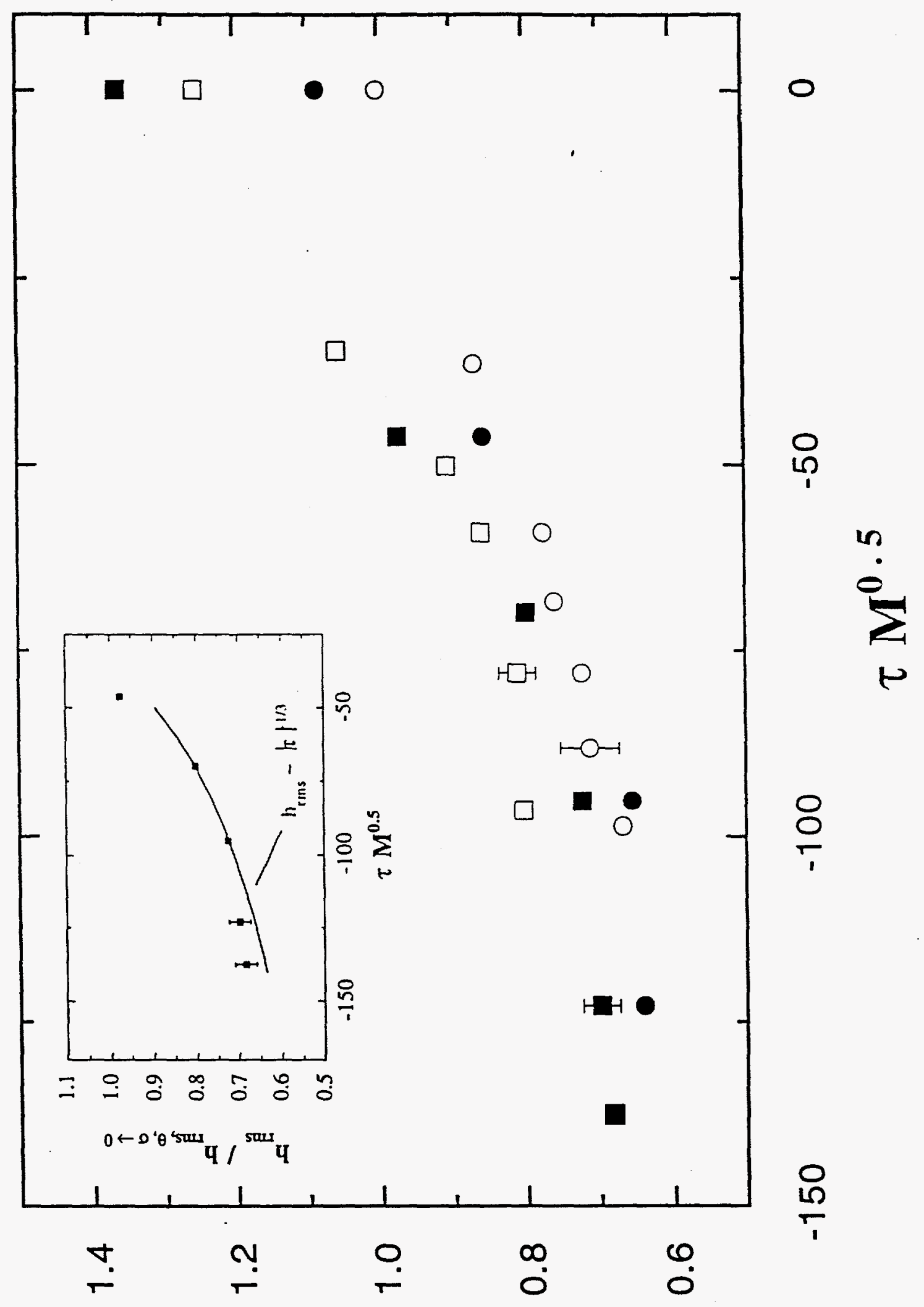

$$
0 \leftarrow \rho^{\circ} \theta \text { 'sux } \mathbf{Y} /{ }^{\text {su. }} \mathbf{Y}
$$




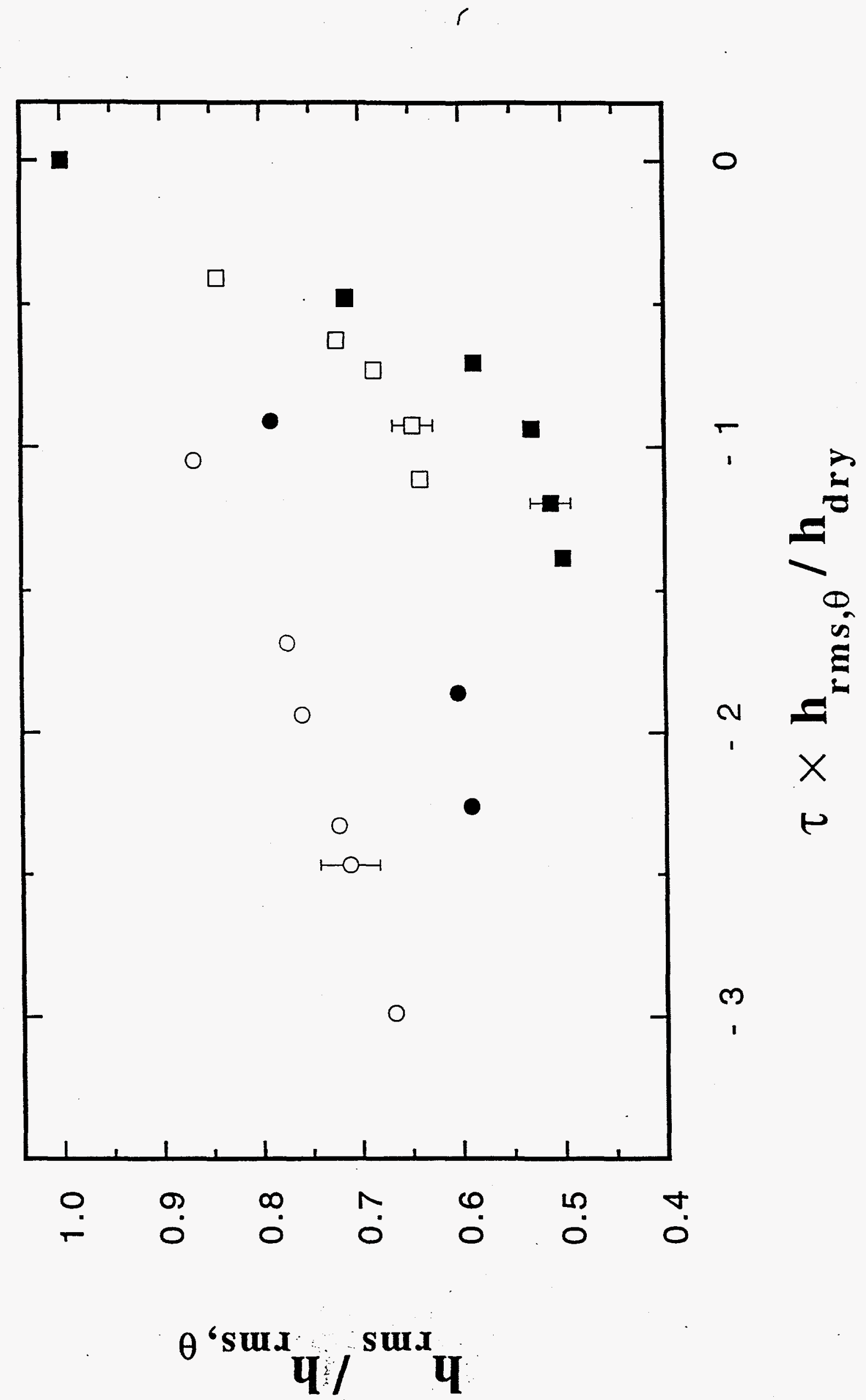




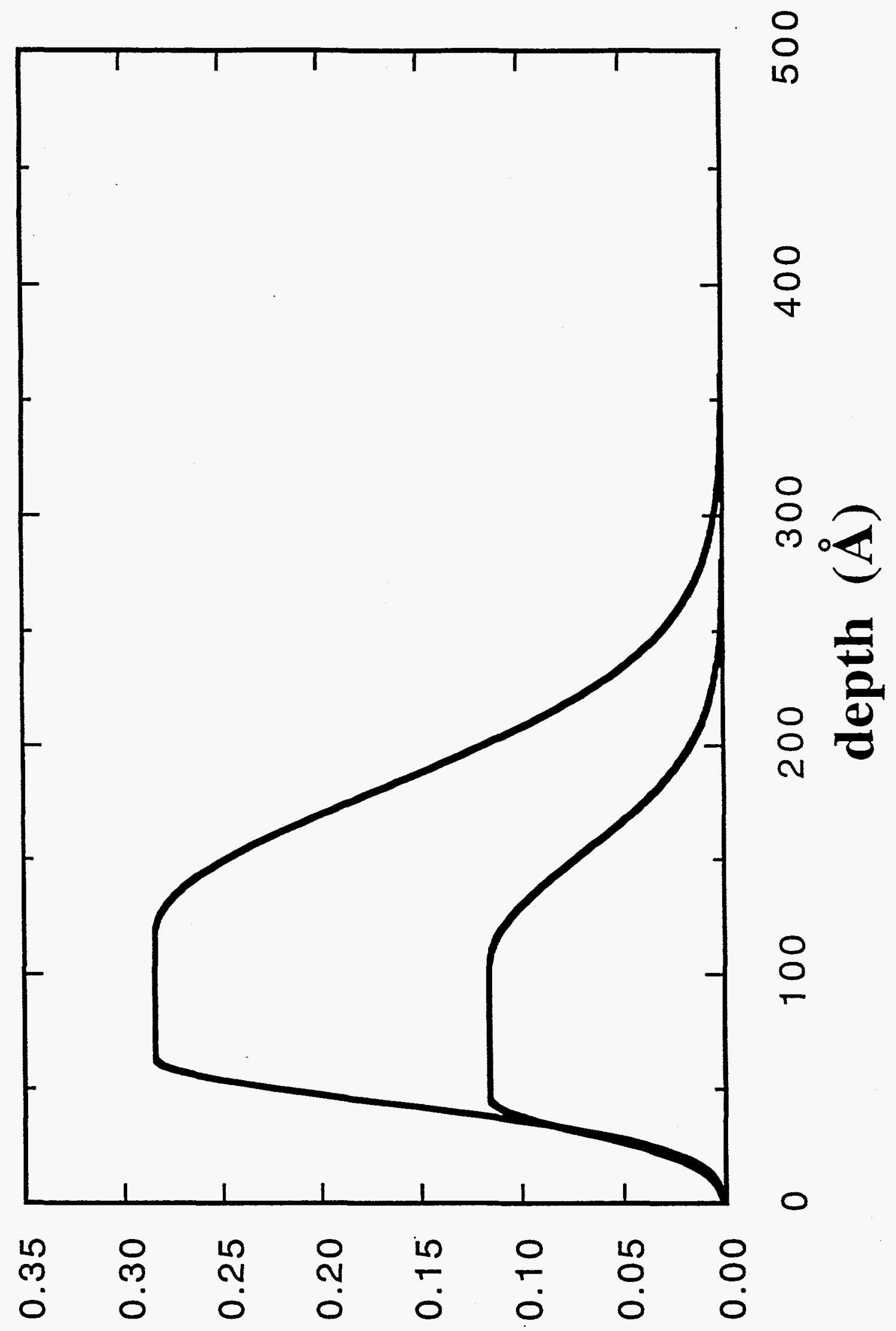

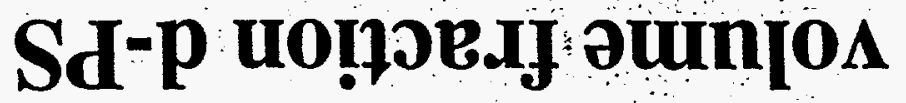




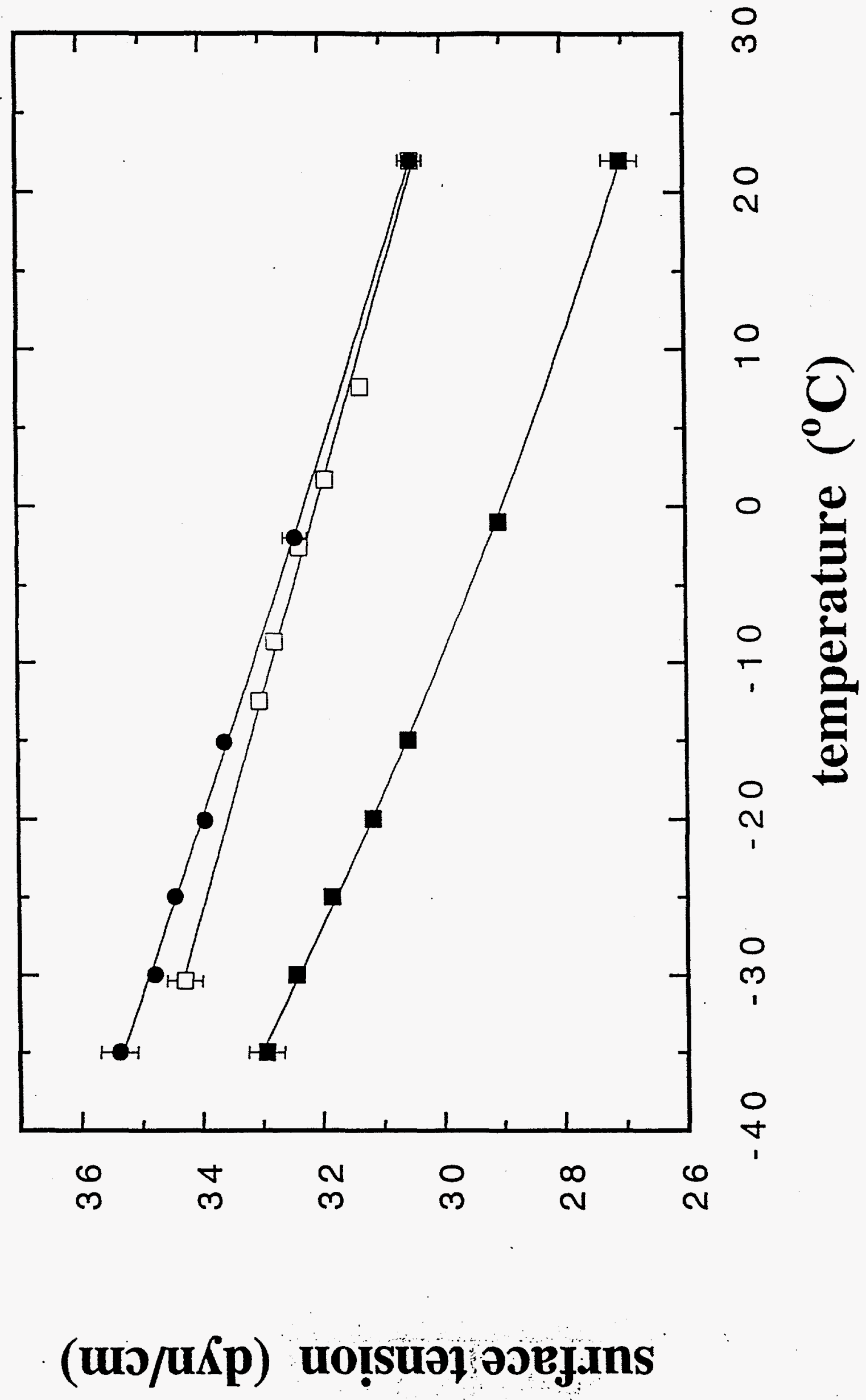




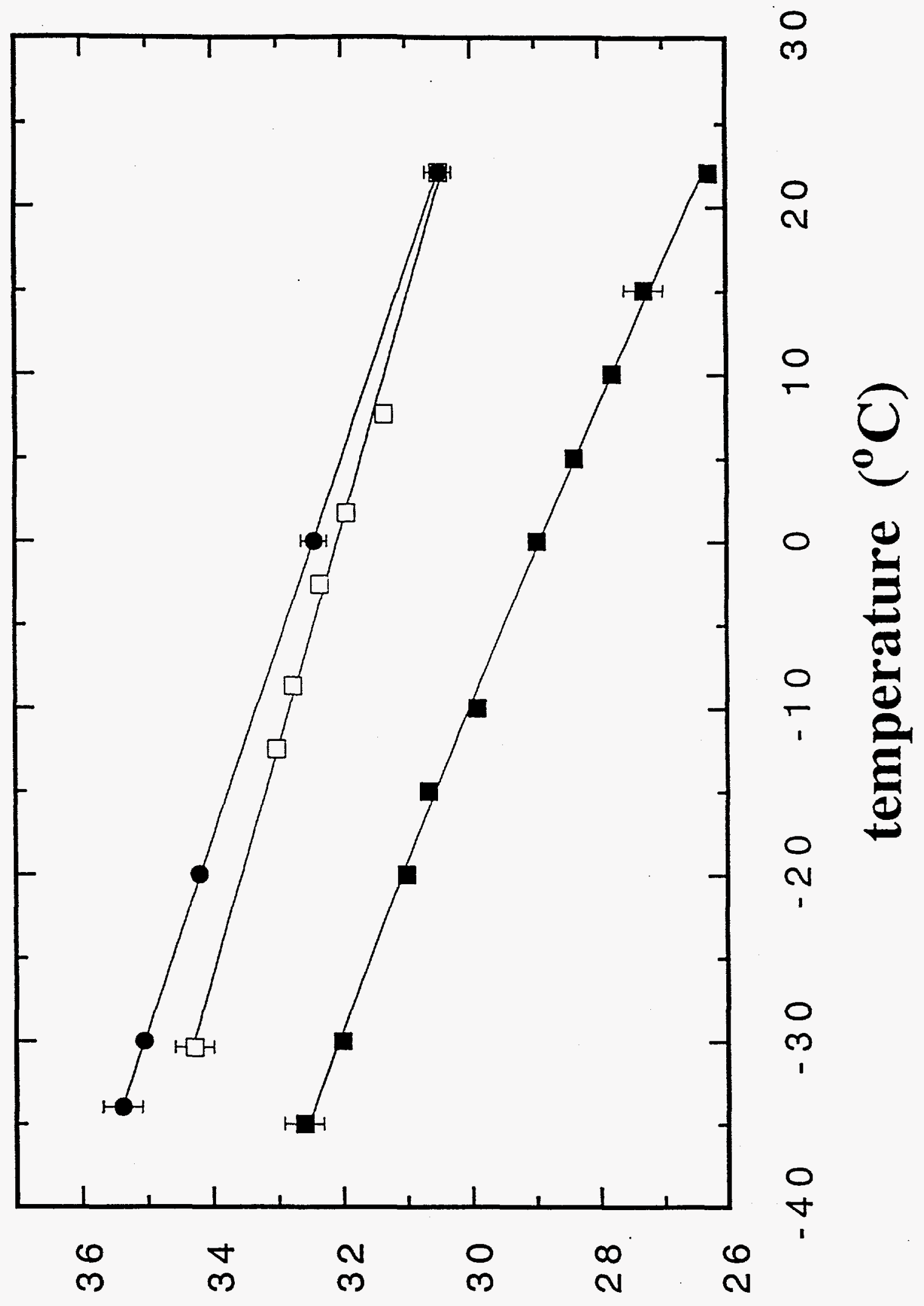

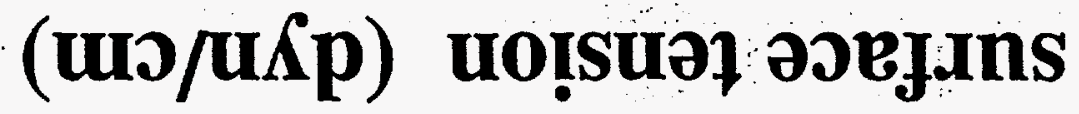




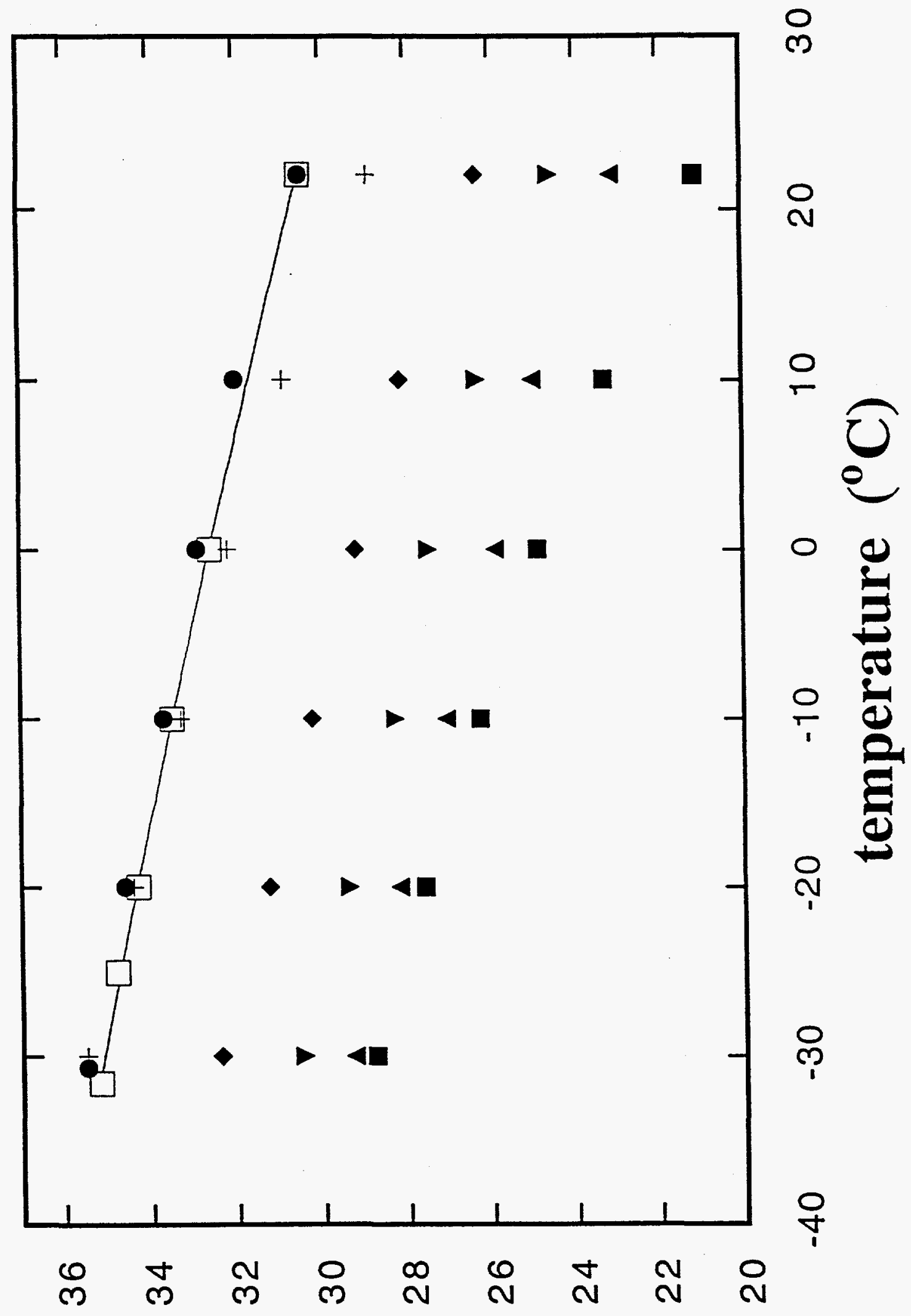

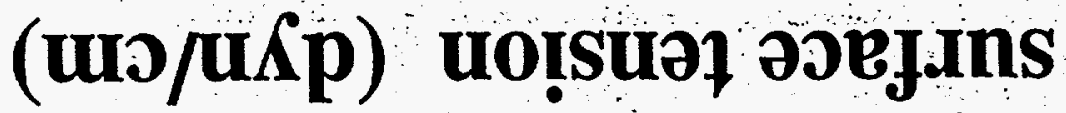




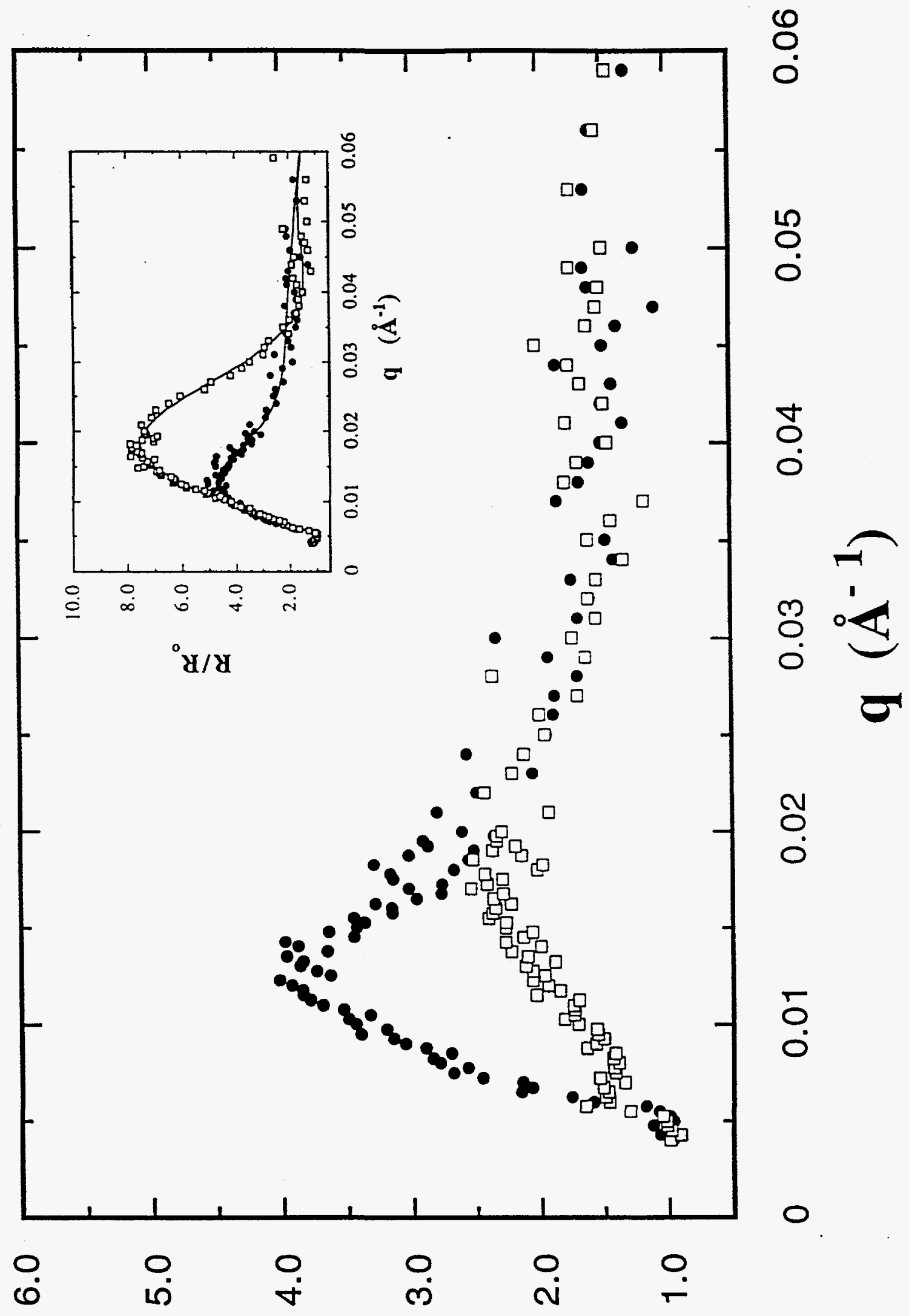

o

y/X 


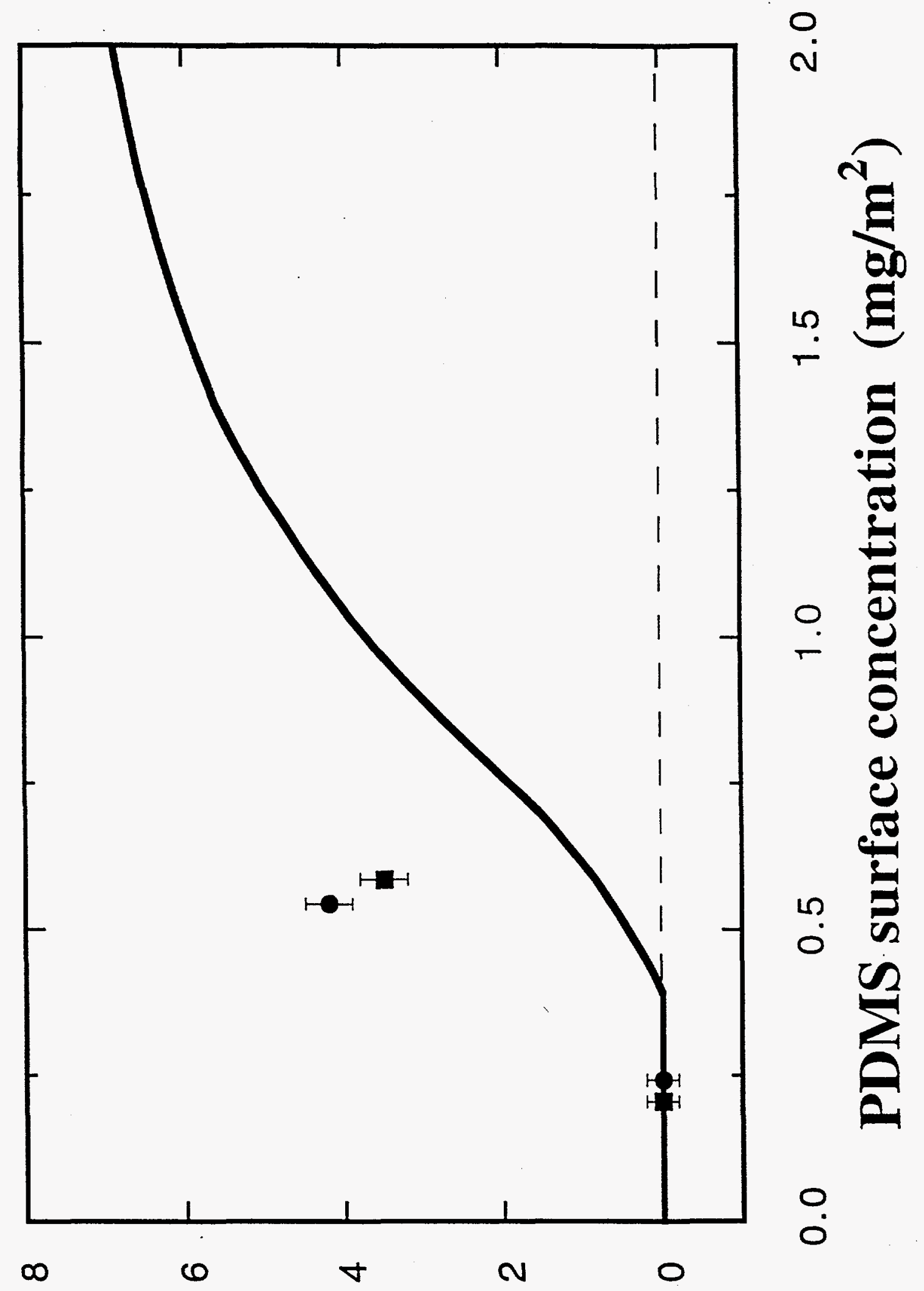

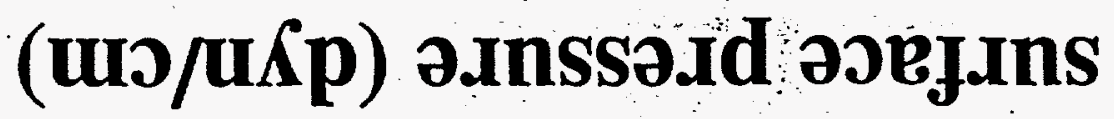




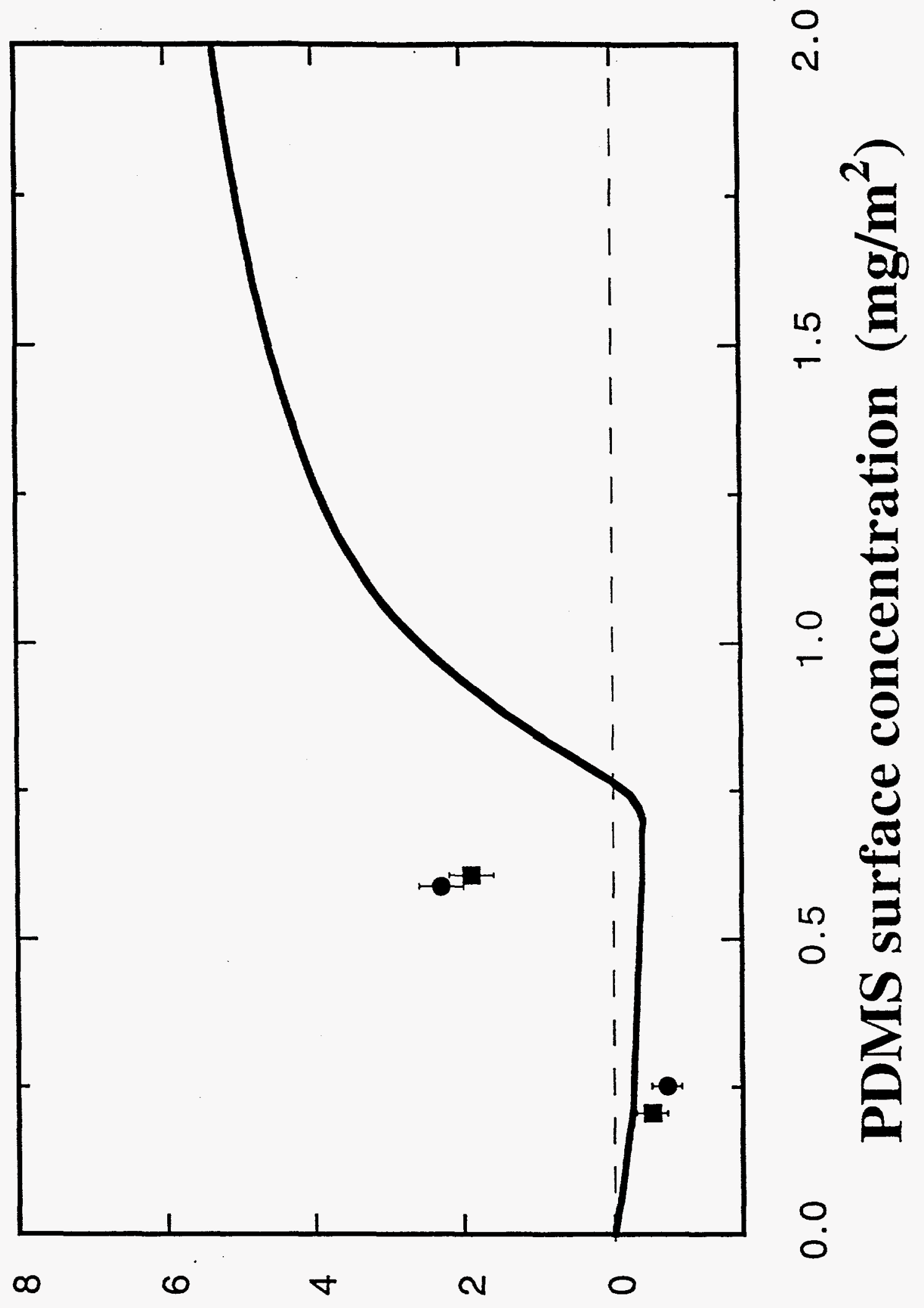

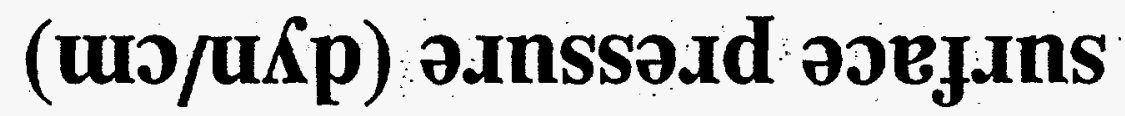




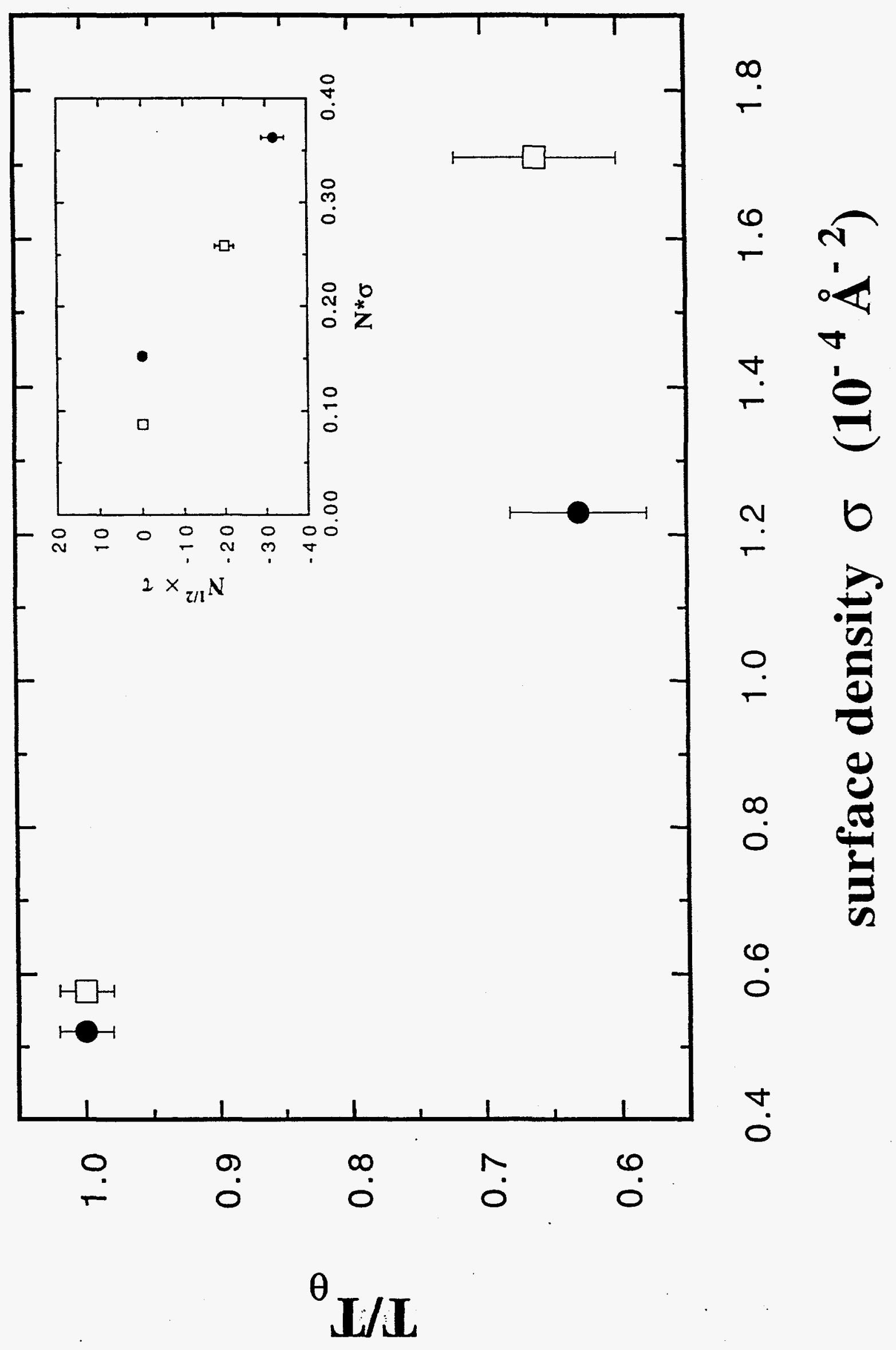




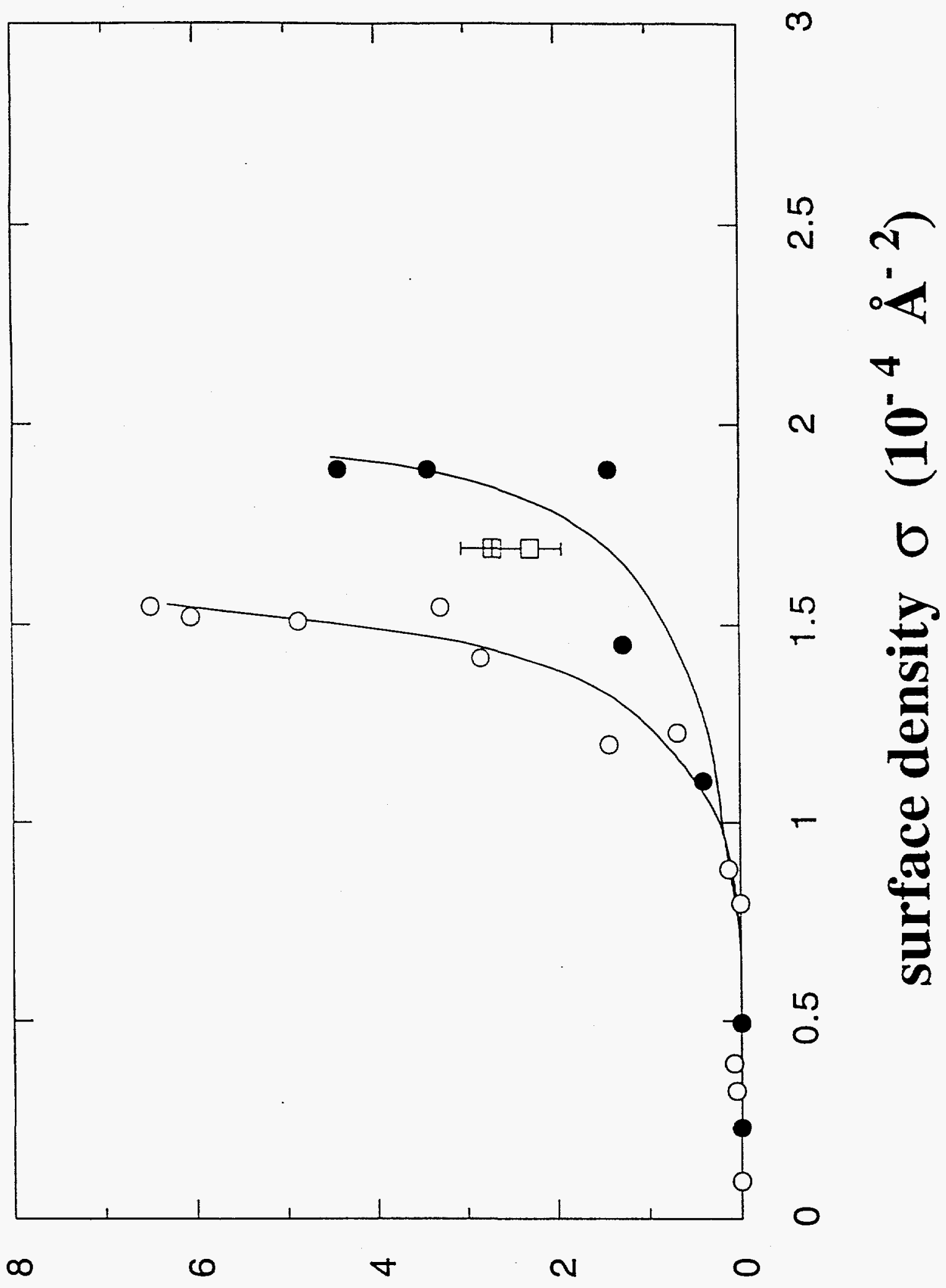

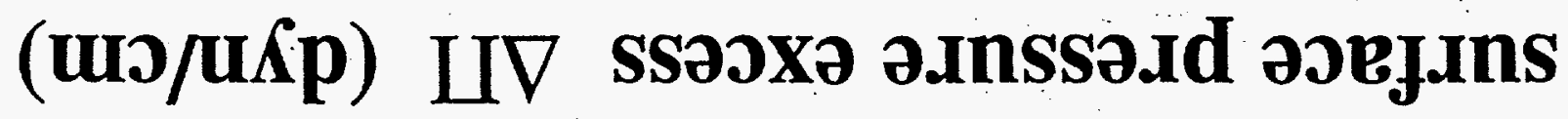




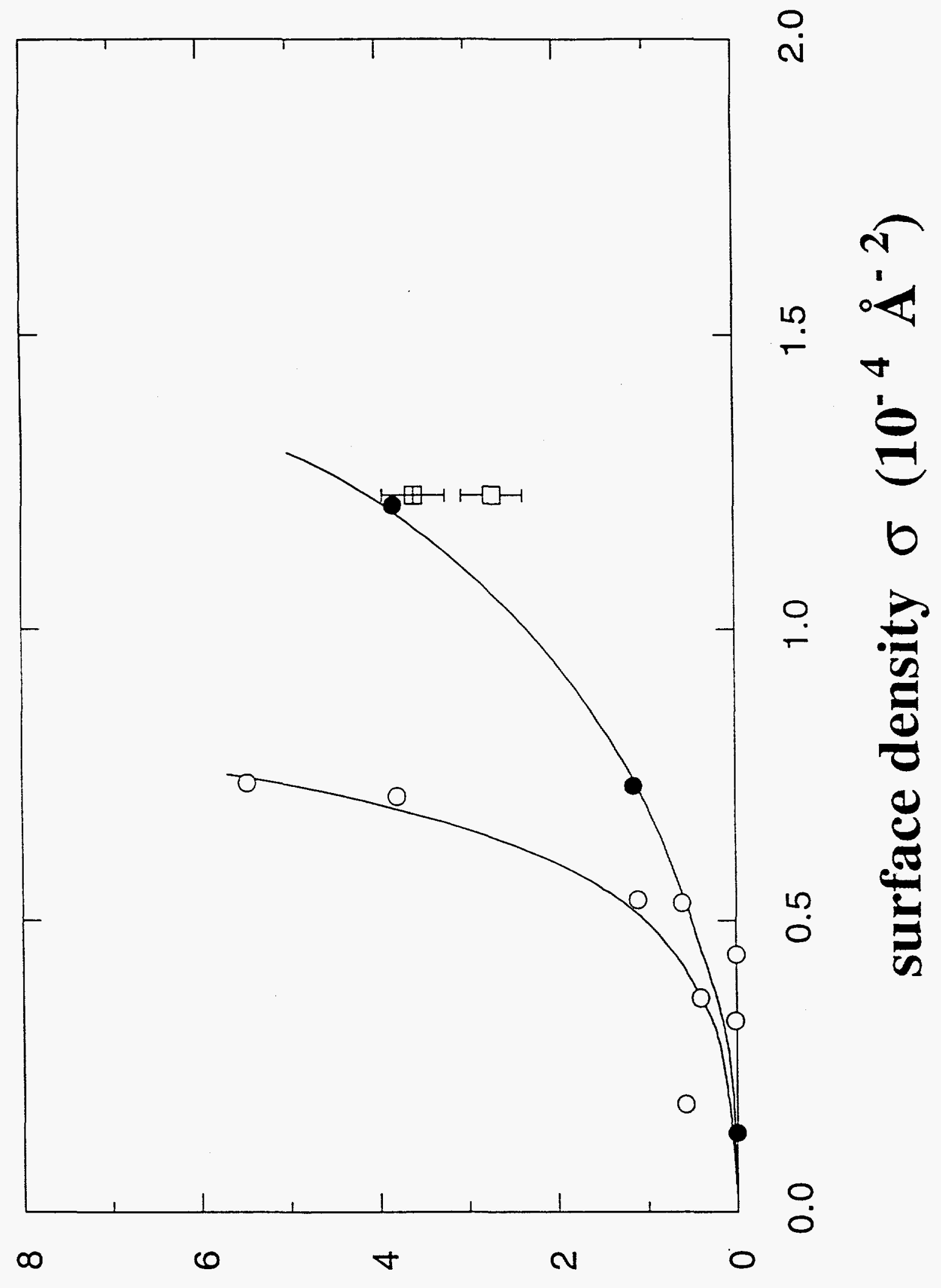

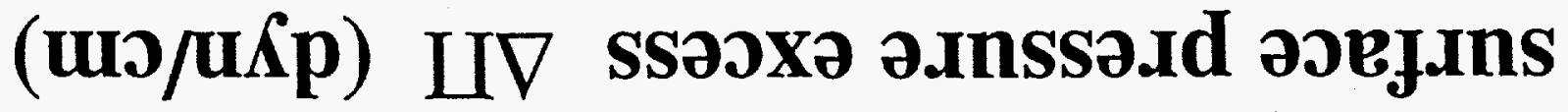

